\title{
Filamentation in the RTP tokamak plasma
}

\author{
M N A Beurskens ${ }^{1}$, N J Lopes Cardozo, E R Arends, C J Barth and \\ H J van der Meiden
}

FOM-Instituut voor Plasmafysica 'Rijnhuizen', Association Euratom-FOM, Trilateral Euregio Cluster, PO Box 1207, 3430 BE Nieuwegein, The Netherlands

E-mail: marc.beurskens@jet.uk

Received 23 February 2000, in final form 4 October 2000

\begin{abstract}
Experimental data are presented showing filamentation of the Rijnhuizen tokamak project (RTP) plasma. These filaments are only resolved by the highresolution double pulse Thomson scattering diagnostic, and appear as multiple peaks in the $T_{\mathrm{e}}$ profile with a typical width of 5-10 $\mathrm{mm}$ and an amplitude as high as $1 \mathrm{keV}$ in a $2 \mathrm{keV}$ ambient plasma. This paper shows the occurrence of filaments under various plasma conditions. It shows that filaments are statistically significant plasma physical phenomena. A parameter study shows a weak dependence of their amplitude on $q_{\mathrm{a}}$, whereas a strong inverse dependence on plasma density has been found. It takes filaments several milliseconds to develop after the switch-on of electron cyclotron heating; they are wiped out by a sawtooth crash and take only a few hundred microseconds to reappear after such a crash. They mainly occur in the centre of additionally heated plasmas by means of electron cyclotron heating, but have also been observed in transiently heated plasmas and off-axis in non-centrally heated plasmas. Finally, two interpretations of filament topology are tested by means of three experiments. It turns out that the interpretation of filaments as independent closed tube-like structures seems to best fit the RTP data.
\end{abstract}

\section{Introduction}

The tokamak is the most common type of machine used in thermonuclear research to confine a hot plasma by magnetic fields. Its beneficial properties of plasma confinement derive from the magnetic topology: in an idealized description the magnetic field lines form nested toroidal surfaces. In this topology, transport of heat or particles in the direction perpendicular to those surfaces is only possible through collisions (neo-classical transport), or through the action of fluctuating electric fields.

However, the field line equations have the mathematical property that surfaces on which the field line winding ratio $(q)$ is rational are topologically unstable [1]. The generic topology

1 Current address: UKAEA JET, Abingdon, Oxfordshire, OX14 3EA, UK. 

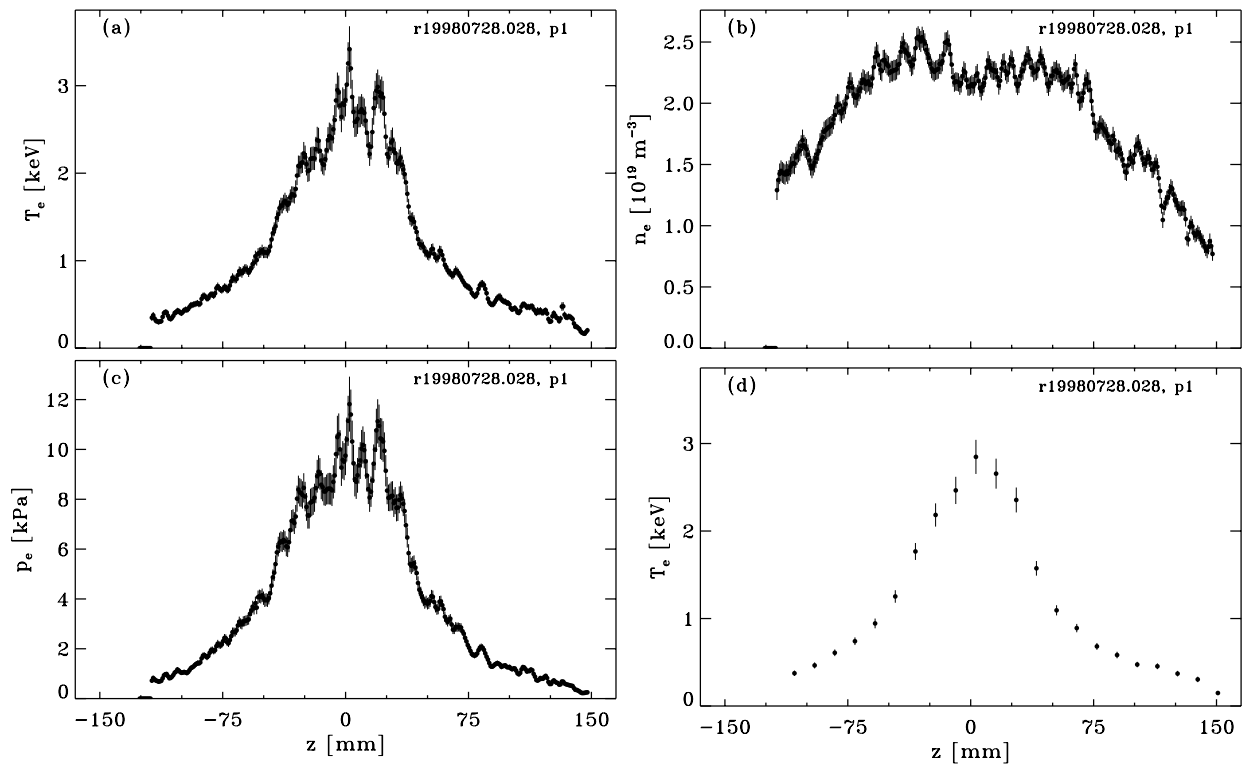

Figure 1. Example of a high-resolution TS measurement: (a) $T_{\mathrm{e}}$, (b) $n_{\mathrm{e}}$ and (c) $p_{\mathrm{e}}$ profile of a central ECH plasma with $q_{\mathrm{a}}=6.2$. The filaments are much more pronounced in $T_{\mathrm{e}}$ and $p_{\mathrm{e}}$ than in $n_{\mathrm{e}}$. (d) The resolution of the RTP ECE-imaging diagnostic $(1.3 \mathrm{~cm}$ FWHM) can be simulated by binning data points from the TS $T_{\mathrm{e}}$ profile shown in (a). Clearly, after binning no filaments are resolved anymore.

of the magnetic field in a tokamak should be a mix of good surfaces, chains of magnetic islands, and regions with chaotic field lines.

Experimental observations of magnetic islands are virtually limited to the very large modes with poloidal mode numbers $m=1$ (the sawtooth pre- and post-cursors) and $m=2$ precursors to the major disruption. Such islands can reach a size of $20 \%$ of the minor radius or more, and are easily detected with several diagnostics, e.g. [2-4]. The evidence of the occurrence of small magnetic islands is much scarcer. Observations of an $m=3, n=2$ mode have been reported from several experiments $[5,6]$, and recently the neo-classical tearing modes have been observed in, e.g., [7].

However, to affect electron transport, even much smaller islands with a size comparable to the banana width, i.e. typically 1-10 mm, can be important. Such islands are very difficult to diagnose. There is no experimental proof that magnetic perturbations do not contribute to electron heat transport. Evidence in favour of this contribution is also scarce. Measurements of fluctuations of the magnetic field in Tore Supra, using the cross-polarization scattering of microwaves, showed fluctuations at the level $\tilde{B}_{\phi} / B_{\phi}>10^{-5}$ with a broad band frequency spectrum [8,9]. It was estimated that these fluctuations could be responsible for the observed electron heat transport. At TEXTOR, a study of the confinement of $30 \mathrm{MeV}$ runaway electrons estimated the scale size of magnetic modes in the range of $0.5 \mathrm{~cm}$ in ohmic plasmas, increasing to several centimetres with high power heating [10].

Other evidence for a broken magnetic topology was obtained at the Rijnhuizen Tokamak Project (RTP). Measurements with an ultrahigh-resolution Thomson scattering (TS) system [11], revealed that the $T_{\mathrm{e}}$ profile shows multiple peaks under intense local electron heating with electron cyclotron heating $(\mathrm{ECH})$. These were interpreted as hot 'filaments', i.e. closed flux tubes with an $m / n=1$ structure $[12,13]$. Figure 1 gives a typical example. The typical 
width of the filaments is 5-10 mm, and they are much more pronounced on $T_{\mathrm{e}}$ than on $n_{\mathrm{e}}$. The occurrence of filaments appeared to be limited to the region inside the $q=1$ surface, and a coarse estimation yielded a lifetime of hundreds of microseconds. The steep gradients in the filaments as well as their life time could be understood by assuming that the confinement inside a filament is very good, corresponding to a thermal diffusivity near the neo-classical value.

A similar observation was reported from TEXT [14], where under ECH strong spatial variations of $T_{\mathrm{e}}$ were observed with electron cyclotron emission (ECE) spectroscopy, in the central region of the plasma. Using the high time resolution of the ECE, it was shown that the structures have an $m=n=1$ periodicity. Based on the TEXT results, Porcelli et al [16] designed a model which qualitatively describes the peaks in the TEXT $T_{\text {e }}$ profile. His model describes a deformation of the $m=1$ sawtooth precursor in ECH plasmas, such that sharp $T_{\mathrm{e}}$ gradients occur in the central region. These manifest as rings with higher temperature.

Mirnov [15] shows that positive magnetic islands, i.e. islands with a higher current density than the ambient plasma, can occur in regions with low magnetic shear. These positive islands show similarity with the RTP interpretation of plasma filaments as separate current carrying flux tubes $[12,13]$.

The observations of hot filaments in RTP raised many questions:

- Are the measurements statistically significant?

- Are the observed peaks in the $T_{\mathrm{e}}$ profile the cross section of toroidally extended structures, or are they local temperature fluctuations?

- If they are spatial structures, are they toroidally closed structures? Or could the very local heating of a bundle of field lines in a chaotic region give rise to similar observations? What is the topology of filaments?

- How do filaments depend on plasma density and current?

- Are the filaments ECH induced or is the ECH only highlighting pre-existing filaments?

- Are there observations of filaments outside the central, $q=1$ region?

- How do filaments behave in a sawtooth crash?

In this paper we address those questions. Since the first observations of hot filaments in RTP the following steps have been made: the RTP TS diagnostic has been upgraded from single pulse to double pulse [6]. This allows a coarse study of the dynamics of filaments, and can, for example, bring out what happens to filaments during a sawtooth crash. An extensive study has been made of the spatial resolution and experimental errors of the new TS diagnostic, allowing a meaningful statistical analysis of the measurements [17]. An extended set of measurements of filamented $T_{\mathrm{e}}$ profiles has been made, in a variety of plasma conditions.

The structure of this paper is as follows. In section 2 we briefly describe the experimental set-up of the high-resolution $T_{\mathrm{e}}$ measurements. In section 3 , the statistical significance of the observed structures in $T_{\mathrm{e}}$ is investigated. In section 4 we address the question of whether filaments are spatial structures or local, temporal fluctuations. In section 5 the dependence of filaments on plasma current and electron density is studied. Section 6 presents an experiment in which the time scale of filament creation upon ECH switch-on is determined. Section 7 answers the questions of whether (1) filaments occur outside the $q \leqslant 1$ region and (2) whether filaments are ECH specific. In section 8 the effect of sawtooth crashes on filaments is presented and section 9 shows three experiments that address the question of the topology of filaments.

Thus, we come to a comprehensive description of the phenomenology of filamentation. In section 10, the discussion concentrates on a number of plasma physical aspects of filamentation such as the magnetic topology of filaments, the current density perturbation in a filament and the energy balance in a filament. Finally, the importance of filamentation for the understanding of transport in tokamak plasmas is addressed. 


\section{High-resolution measurements}

The Rijnhuizen Tokamak Project (RTP; $R / a=0.72 / 0.165 \mathrm{~m}, B_{\mathrm{T}} \leqslant 2.5 \mathrm{~T}, I_{\mathrm{p}} \leqslant 150 \mathrm{kA}$, $q_{\mathrm{a}} \geqslant 2$ ) is equipped with high-power additional electron heating and an extensive set of highresolution diagnostics [18]. A $350 \mathrm{~kW}, 110 \mathrm{GHz} \mathrm{ECH}$ system can deposit heat in a very localized area ( $\sim 10 \%$ of the minor radius) in the plasma. The $\mathrm{ECH}$ can be the dominant heat source, exceeding the ohmic power by an order of magnitude. In an ECH plasma, $T_{\mathrm{e}}$ is up to $4 \mathrm{keV}$ at $n_{\mathrm{e}}=2 \times 10^{19} \mathrm{~m}^{-3}$, while in a typical ohmic plasma $T_{\mathrm{e}}$ reaches $0.7 \mathrm{keV}$. The highresolution diagnostics include a 20-channel ECE radiometer measuring $T_{\mathrm{e}}$ along a horizontal line, a 16-channel ECE imaging (ECE-I) diagnostic measuring $T_{\mathrm{e}}$ along a vertical line, a 19channel interferometer, and an 80-channel five-camera soft $\mathrm{x}$-ray tomographic system. Of these, the ECE systems offer the best spatial resolution of $\geqslant 1.3 \mathrm{~cm}$, i.e. $8 \%$ of the minor radius.

Crucial for the present paper is the double pulse multiposition TS diagnostic [6]. It measures $T_{\mathrm{e}}$ and $n_{\mathrm{e}}$, in a snapshot of $40 \mathrm{~ns}$, at 350 points along a vertical chord of $300 \mathrm{~mm}$. Its spatial resolution is $3 \mathrm{~mm}$ full width at half maximum (FWHM), i.e. $2 \%$ of the minor radius. It measures two profiles per discharge with a tunable time separation of 20-800 $\mu$ s, enabling a study of the dynamics of small-scale structures in the plasma. Its statistical relative error is 3-5\% of $T_{\mathrm{e}}$ and $2-4 \%$ of $n_{\mathrm{e}}$ for $T_{\mathrm{e}}$ in the range of $50 \mathrm{eV}-6 \mathrm{keV}$, and $n_{\mathrm{e}}=5 \times 10^{19} \mathrm{~m}^{-3}$ [17]. The systematic error on the values of $T_{\mathrm{e}}$ and $n_{\mathrm{e}}$ may be larger, but this does not influence the study of small-scale structures [17].

At RTP, the diagnostic with the second best resolution is the ECE-I diagnostic, but its spatial resolution of $1.3 \mathrm{~cm}$ FWHM is insufficient to observe the filaments, as is illustrated in figure 1. Moreover, because ECE-I and ECH at RTP both operate at the second harmonic of the electron cyclotron frequency, central ECE-I measurements are not possible during central $\mathrm{ECH}$.

\section{Statistical significance of filaments}

The first question to be addressed concerning the observations of filaments, such as shown in figure 1 , is whether they are a plasma physical phenomenon or a diagnostic artefact. The evidence that they are a plasma physical phenomenon consists of the following. In this paper results are presented:

(1) showing that the amplitude of the filaments depends on plasma parameters such as density and plasma current (section 5);

(2) showing that in the initial period after switching on the ECH, it takes several milliseconds for the filaments to form, while $T_{\mathrm{e}}$ and $n_{\mathrm{e}}$ reach their equilibrium values much faster (section 6);

(3) showing that filaments that were still there just before a sawtooth instability, have disappeared directly after the sawtooth collapse (section 8);

(4) showing that filaments are periodic structures that reappear in the TS profile after a full rotation of the plasma (section 9).

Each of these observations is very difficult to interpret when the filaments are a diagnostic artefact.

In addition, in this section a statistical test is performed of the presence of filaments in an ensemble of 22 similar discharges, taken at one day. All three $T_{\mathrm{e}}, n_{\mathrm{e}}$ and $p_{\mathrm{e}}$ profiles are analysed. The test uses an estimation of the statistical errors on the three parameters, which is 

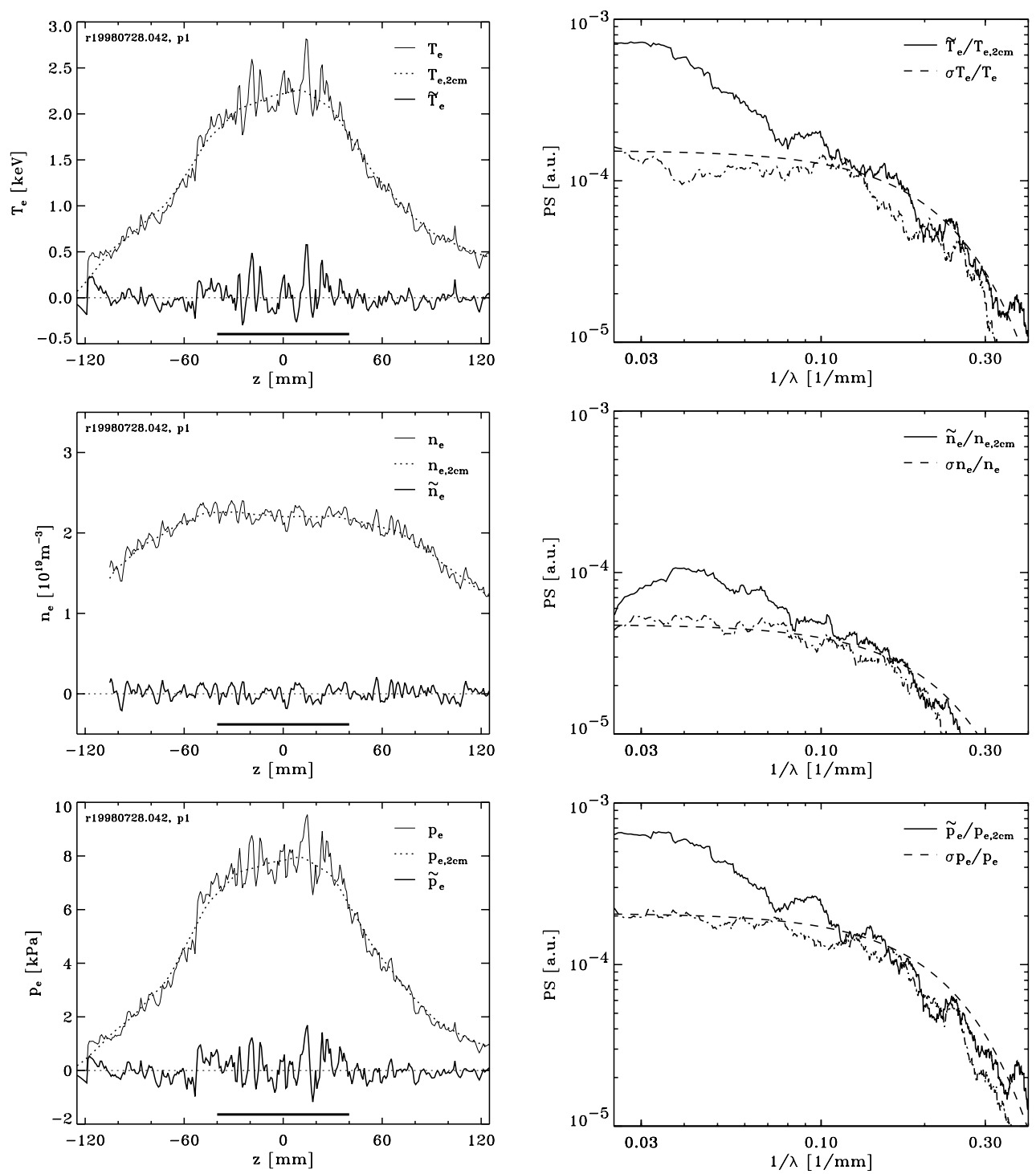

Figure 2. Typical profiles of $T_{\mathrm{e}}$ (thin line), $\tilde{T}_{\mathrm{e}}$ (thick line), $n_{\mathrm{e}}, \tilde{n}_{\mathrm{e}}, p_{\mathrm{e}}$ and $\tilde{p}_{\mathrm{e}}$ of a plasma with central ECH The fluctuation profiles are obtained by subtracting a smooth profile $\left(T_{\mathrm{e}, 2 \mathrm{~cm}}, n_{\mathrm{e}, 2 \mathrm{~cm}}\right.$ and $\left.p_{\mathrm{e}, 2 \mathrm{~cm}}\right)$ from the measured profile (see text). The horizontal bar drawn from $z=[-40,40] \mathrm{mm}$, shows the area to which the power spectrum analysis is restricted.

Figure 3. Power spectrum (for 22 discharges) of $\tilde{T}_{\mathrm{e}} / T_{\mathrm{e}, 2 \mathrm{~cm}}, \tilde{n}_{\mathrm{e}} / n_{\mathrm{e}, 2 \mathrm{~cm}}$ and $\tilde{p}_{\mathrm{e}} / p_{\mathrm{e}, 2 \mathrm{~cm}}$ (drawn) compared to the power spectra of simulated noise $\sigma_{T_{\mathrm{e}}} / T_{\mathrm{e}}, \sigma_{n_{\mathrm{e}}} / n_{\mathrm{e}}$ and $\sigma_{p_{\mathrm{e}}} / p_{\mathrm{e}}$ (dash-dot). Overplotted (dashed) is the noise characteristic of a Gaussian filter with 2.4 pixels FWHM. The simulated noise comes out somewhat lower.

the result of a detailed error breakdown of the TS measurements [17]. The 22 discharges had central ECH, $q_{\mathrm{a}} \approx 4.6$ and $n_{\mathrm{e}}(0) \approx 2.4 \times 10^{19} \mathrm{~m}^{-3}$.

Figure 2 shows the profiles of a typical example taken from the test series of 22 discharges. For this data set, power spectra of the $T_{\mathrm{e}}, n_{\mathrm{e}}$ and $p_{\mathrm{e}}$ profiles are calculated and compared to the 'known' noise spectrum. To suppress spurious contributions of the global profile shape 
and parts of the profile that do not show filamentation, (i) the analysis is restricted to the $z$ range $[-40,40] \mathrm{mm}$, and (ii) a smooth profile is subtracted from the measured profile. The smooth profiles, $T_{\mathrm{e}, 2 \mathrm{~cm}}, n_{\mathrm{e}, 2 \mathrm{~cm}}$ and $p_{\mathrm{e}, 2 \mathrm{~cm}}$, are obtained by convolving the measured profile with a Gaussian, with $20 \mathrm{~mm}$ FWHM. The 22 thus obtained profiles of the central part of $\tilde{T}_{\mathrm{e}}=T_{\mathrm{e}}-T_{\mathrm{e}, 2 \mathrm{~cm}}$, are normalized to $T_{\mathrm{e}, 2 \mathrm{~cm}}$, and merged to form one long array (the same is done for $n_{\mathrm{e}}$ and $p_{\mathrm{e}}$ ). The power spectrum of this array is shown in figure 3. This spectrum is compared to the simulated noise spectrum. Note that the decay of both the noise and the measurement at $1 / \lambda>0.2 \mathrm{~mm}^{-1}$ is due to the convolution with the instrument function, i.e. a Gaussian with 2.4 pixel FWHM. The fact that measured spectrum and noise characteristic in this range fall on top of each other, shows that the noise level is correctly estimated.

Figure 3 shows that the measured spectra are well above the noise below the cut-off due to the instrument function at $1 / \lambda=0.16 \mathrm{~mm}^{-1}$. Thus, all three $T_{\mathrm{e}}, n_{\mathrm{e}}$ and $p_{\mathrm{e}}$ have significant structures. However, $n_{\mathrm{e}}$ structures have almost one order of magnitude smaller amplitude than $T_{\mathrm{e}}$ and $p_{\mathrm{e}}$ structures.

The peak in the spectrum represents the average spacing of the filaments, which for $1 / \lambda=0.02-0.05$ corresponds to $2-5 \mathrm{~cm}$, or one to four filaments in the central region. The higher frequencies in the spectrum are generated by the irregular occurrence of the filaments, and the fact that their half-width is smaller than their interspacing. The fact that the spectrum does approach the noise level around $1 / \lambda=0.1$ indicates that filaments with a width smaller than $5 \mathrm{~mm}$ are rare, but the proximity to the resolving power of the diagnostic prohibits any strong conclusion here.

In conclusion, the spectral analysis supports the hypothesis that in the measured $T_{\mathrm{e}}$ profiles of centrally heated discharges in RTP, statistically significant filaments are observed with the following properties:

(1) typical occurrence: several filaments in the region $[-40,40] \mathrm{mm}$;

(2) typical radial width larger than $5 \mathrm{~mm}$, and smaller than the average half interspacing of about $20 \mathrm{~mm}$.

This agrees with the conclusions of a visual inspection of a series of profiles, in which typically one to four filaments with a width of 5-10 $\mathrm{mm}$ are observed.

\section{What are filaments?}

Interpreting the filaments as a plasma physical phenomenon, the first question to be addressed is: what is their shape in three-dimensional space. We consider a few possibilities, between which we shall try to distinguish on the basis of experimental material as well as theoretical considerations:

(1) What appear as filaments in the $T_{\mathrm{e}}$ profile are fast, local $T_{\mathrm{e}}$ fluctuations which are recorded in the $40 \mathrm{~ns}$ snapshot of the TS measurement.

(2) A filament is a bunch of neighboring field lines which cross the $\mathrm{ECH}$ heating zone but do not form a topological entity such as a magnetic island.

(3) A filament is (part of) a closed, toroidal magnetic structure such as a magnetic island.

Note that in cases 2 and 3 it is still possible and also likely that the filaments do change with time. However, if spatial structures live for many collision times and local current diffusion times, the spatial structure can be regarded as the essential feature.

In section 9 we show that with double pulse TS, filaments appear only slightly changed after a full period of plasma rotation. This argues against option 1. Further, as was already shown in $[12,13]$, filaments live on for several tenths of milliseconds after ECH is switched 

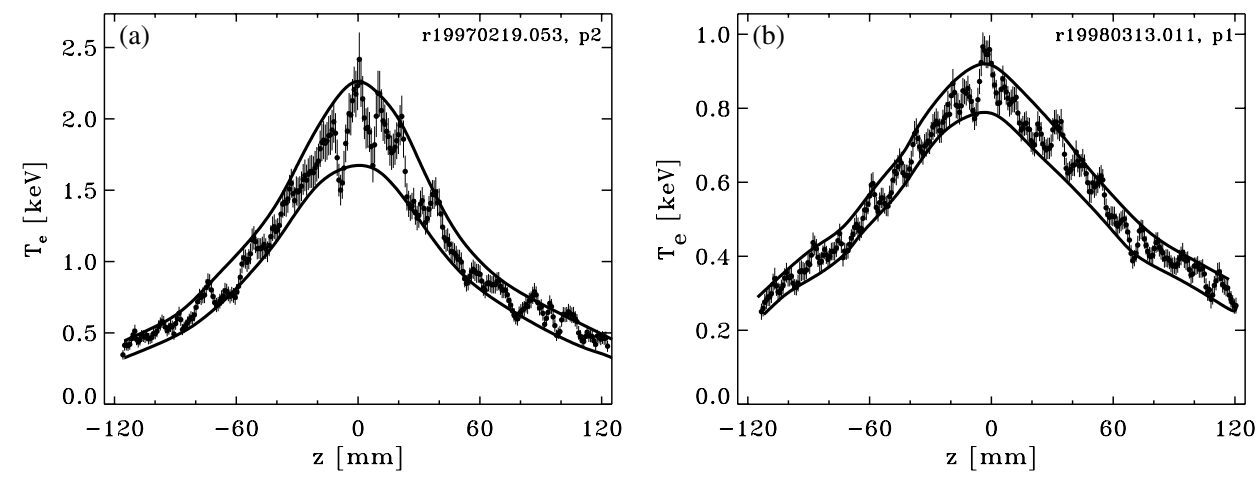

Figure 4. Example of a 'lower' and an 'upper' profile of $T_{\mathrm{e}}$ in (a) an ECH discharge, $n_{\mathrm{e}}(0)=2.0 \times 10^{19} \mathrm{~m}^{-3}, \Theta_{T_{\mathrm{e}}}=650 \mathrm{eV},(\mathrm{b})$ an ohmic discharge, $n_{\mathrm{e}}(0)=2.9 \times 10^{19} \mathrm{~m}^{-3}$, $\Theta_{T_{\mathrm{e}}}=110 \mathrm{eV}$.

off, and this again is difficult to square with option 1. Upon switching on ECH, it even takes milliseconds for the filaments to develop, as is shown in section 6 .

Thus, the filaments must be spatial structures. Option 2 also can be rejected on the basis of the following argument. To highlight a small region in a chaotic sea, a very strong $T_{\mathrm{e}}$ gradient must be sustained along the field lines. Assuming that the typically two to three observed filaments are all the same bunch of 'hot' field lines, a temperature difference of up to $1 \mathrm{keV}$ must be sustained over a distance of two toroidal transits, or $10 \mathrm{~m}$ in RTP. This requires a thermal diffusivity of four orders lower than the classical case. This argument becomes even stronger if the finite time any collection of field line is in the heating zone is taken into consideration. Thus, the filaments can only be understood if they are (part of) closed, toroidal structures like item 3. The topology of these magnetic structures is discussed in section 9 .

\section{Parametric study of filaments in ECH plasmas}

\subsection{A quantifier for filamentation}

In order to study the amplitude of the filaments as a function of plasma parameters $\left(n_{\mathrm{e}}, q_{\mathrm{a}}\right)$ and time, we need a quantifier of the filament amplitude. Since the number of observed filaments as well as their positions differ from shot to shot, such a quantifier cannot be based on an averaging algorithm. We have considered various options, and have chosen for the simplest prescription: the quantifier $\left(\Theta_{T_{\mathrm{e}}}\right)$ is equal to the amplitude of the largest filament in the profile. Other recipes (such as the RMS amplitude of the $T_{\mathrm{e}}$ deviations in a prescribed part of the plasma) fail in the presence of steep gradients due to MHD modes.

To determine its value in a reproducible way, we determine two smooth curves that follow the maxima and minima of the profile, respectively. The filament amplitude is defined as the maximum distance between the curves (see figure 4); the curves are determined by hand. Automation of the procedure failed because the software routines could not reliably distinguish between excursions due to filaments (5-10 mm structures) and those due to steep gradients, as in transport barriers. The manual analysis, however, may introduce some arbitrariness. Therefore, we performed two tests of the reliability of the quantifier. First, we had several researchers work out the same series of measured profiles independently. The differences in results were negligible. Second, we applied the quantifier to two similar series of measurements with central ECH. Both sets have average $q_{\mathrm{a}}=5.9 \pm 0.15, I_{\mathrm{p}}=60 \mathrm{kA}$, and central density 

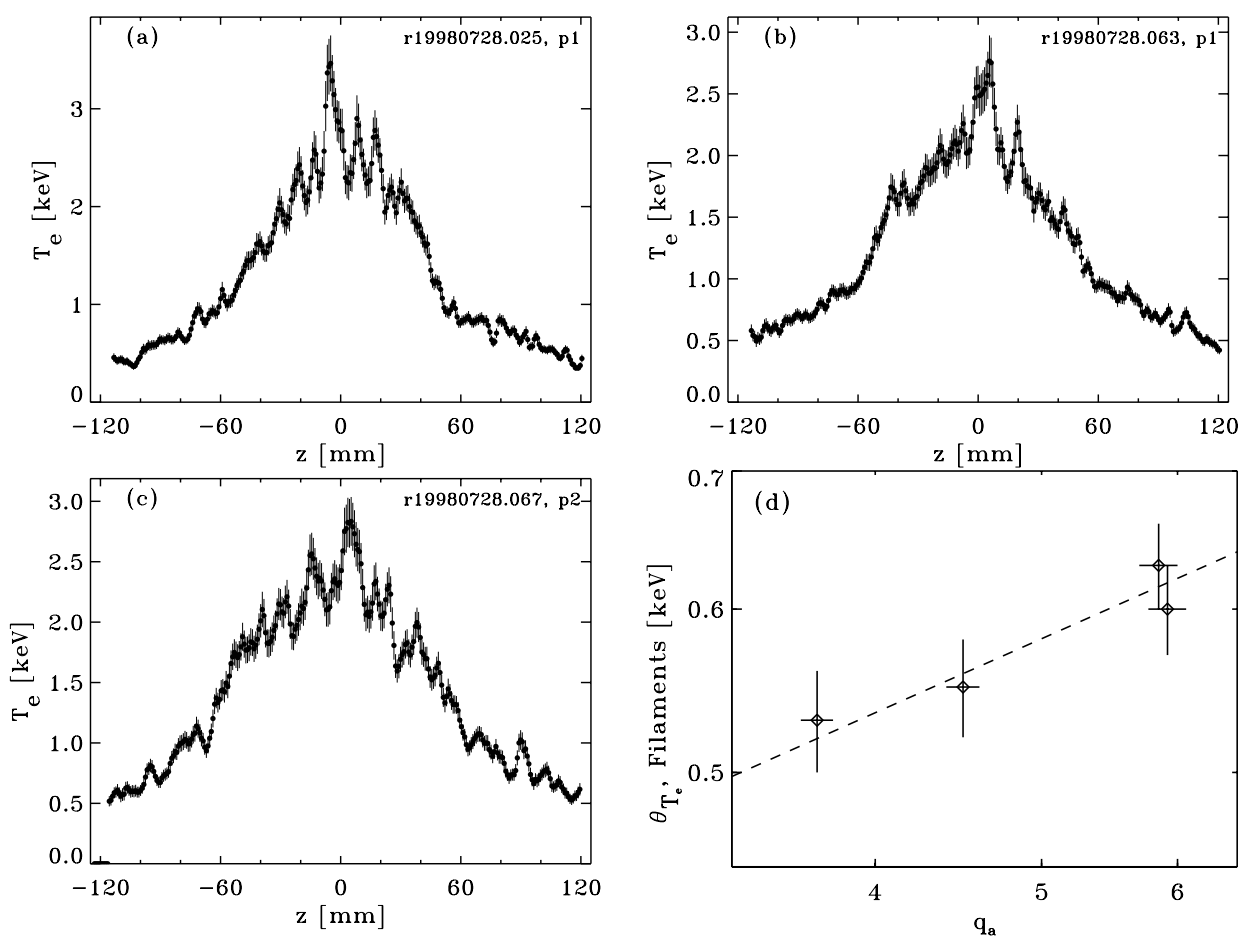

Figure 5. $T_{\mathrm{e}}$ profiles of plasmas with central ECH and different values of $q_{\mathrm{a}}$. (a) $q_{\mathrm{a}}=6.1$, (b) $q_{\mathrm{a}}=4.6$ and (c) $q_{\mathrm{a}}=3.7 . n_{\mathrm{e}}(0) \approx 2.5 \times 10^{19} \mathrm{~m}^{-3}$. (d) Double logarithmic plot of $\Theta_{T_{\mathrm{e}}}$ as a function of $q_{\mathrm{a}}$. The dependence is weak, $\Theta_{T_{\mathrm{e}}} \sim q_{\mathrm{a}}^{0.4}$, and is overplotted with a dashed line. The values of $\Theta_{T_{\mathrm{e}}}$ are an average for four series of discharges: $q_{\mathrm{a}} \approx 5.9$ : two series with 13 and 25 double pulse measurements, $q_{\mathrm{a}} \approx 4.5: 25$ double pulse measurements, and $q_{\mathrm{a}} \approx 3.7$ : nine double pulse measurements.

$n_{\mathrm{e}}(0) \approx 2.1 \times 10^{19} \mathrm{~m}^{-3}$. They were taken more than one year apart. One, taken in February 1997 with 25 discharges, has an average quantifier of $\Theta_{T_{\mathrm{e}}}=590 \pm 30 \mathrm{eV}$, and the other, taken in July 1998 with 13 discharges, has an average quantifier of $\Theta_{T_{\mathrm{e}}}=625 \pm 30 \mathrm{eV}$. Thus, the quantifier gives consistent results for the two data sets, with the same plasma conditions.

In summary, we have defined a quantifier that can be determined reliably and reproducibly. In this paper we use it to determine trends and correlations; we do not use it as an absolute measure of the filament amplitude.

\section{2. $q_{a}$ dependence}

Four series, each containing discharges with similar $q_{\mathrm{a}}$, are used in the comparison. The series have $q_{\mathrm{a}} \approx 3.7,4.6$, and $5.9(2 \times)$, respectively. To vary $q_{\mathrm{a}}$, the plasma current was varied: $I_{\mathrm{p}}=100,80$ and $60 \mathrm{kA}$. The toroidal field $B_{\phi} \approx 2 \mathrm{~T}$, and the plasma density was $n_{\mathrm{e}}(0) \approx 2.1 \times 10^{19} \mathrm{~m}^{-3}$ for all series. Figure 5 shows three examples of $T_{\mathrm{e}}$ profiles for each of the three values of $q_{\mathrm{a}}$. The filament amplitude expressed in average $\Theta_{T_{\mathrm{e}}}$ is plotted in figure 5(d). The figure shows a weak dependence of $\Theta_{T_{\mathrm{e}}}$ on $q_{\mathrm{a}}$. The data are presented in a double logarithmic plot. The fit in the figure shows $\Theta_{T_{\mathrm{e}}} \sim q_{\mathrm{a}}^{0.4}$.

This finding should be considered in the context of two extreme models for the filament amplitude. In the first, we assume that the number of filaments scales with $r_{q=1}^{2}$, and that the 

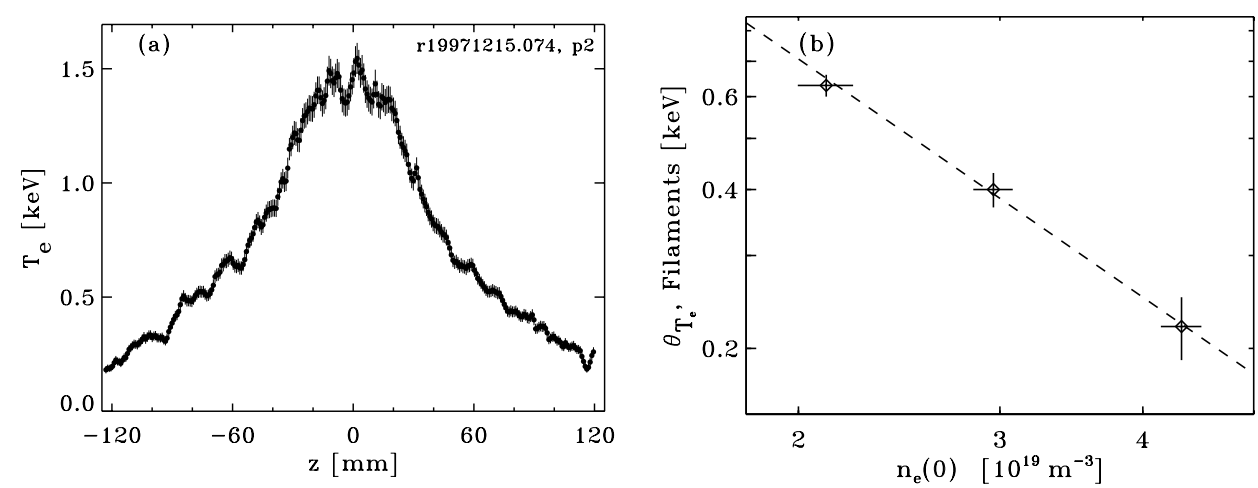

Figure 6. (a) $T_{\mathrm{e}}$ profile of a plasma with central ECH, $q_{\mathrm{a}}=6.1, B_{\phi}=2 \mathrm{~T}, I_{\mathrm{p}}=60 \mathrm{kA}$ and $n_{\mathrm{e}}(0)=4.3 \times 10^{19} \mathrm{~m}^{-3}$. (b) Double logarithmic plot of $\Theta_{T_{\mathrm{e}}}$ as a function of $n_{\mathrm{e}}(0)$. The dependence is very strong, $\Theta_{T_{\mathrm{e}}} \sim n_{\mathrm{e}}^{-1.5}$ indicated by the dashed lines. The average $q_{\mathrm{a}}$ is 5.9 for all three series.

power is distributed over all filaments. This yields: $\Theta_{T_{\mathrm{e}}} \sim q_{\mathrm{a}}^{2}$. The other extreme assumption is that the filament creation is limited to the $\mathrm{ECH}$ deposition region, which is much smaller than the cross section inside $q=1$. In this case the filament amplitude should be independent of $q_{\mathrm{a}}: \Theta_{T_{\mathrm{e}}} \sim q_{\mathrm{a}}^{0}$. The experimentally found $q_{\mathrm{a}}^{0.4}$ relation lies between those two extreme assumptions. It is therefore a plausible result, but at this point there is no quantitative theory to compare it to.

\section{3. $n_{e}$ dependence}

Figure 6 shows a $T_{\mathrm{e}}$ profile of a plasma with central ECH and $n_{\mathrm{e}}(0)=4.3 \times 10^{19} \mathrm{~m}^{-3}, q_{\mathrm{a}}=6.1$, $I_{\mathrm{p}}=60 \mathrm{kA}$. It is clear that it shows much less filamentation then the profile in figure 5(a), which has similar conditions but lower $n_{\mathrm{e}}$.

For three series of discharges at different densities the filamentation quantifier has been determined. The plasma parameters were: $q_{\mathrm{a}} \approx 6.0, I_{\mathrm{p}}=60 \mathrm{kA}, B_{\phi}=2 \mathrm{~T}$, and the three densities: $n_{\mathrm{e}}(0)=2.1,2.9$ and $4.3 \times 10^{19} \mathrm{~m}^{-3}$. Figure $6(\mathrm{~b})$ shows the decrease in filament amplitude with increasing density, in a double logarithmic plot. The dependence is described by $n_{\mathrm{e}}^{-1.5}$. This density scaling is close to that expected when the transport inside a filament is neo-classical. The power balance of filaments is discussed in more detail in section 10 .

\section{Time scale of filament creation}

Figure 7 shows a sequence of single pulse $T_{\mathrm{e}}$ profiles measured after $\mathrm{ECH}$ switch-on in well reproducing discharges. The profiles are taken $0.85,1.85,3.85$, and $5.35 \mathrm{~ms}$ after switchon. The central $T_{\mathrm{e}}$ rises and the figure shows that the filament amplitude increases. The line integrated density is $5 \times 10^{18} \mathrm{~m}^{-2}$ for all discharges, but the central density decreases from $2.8 \times 10^{19} \mathrm{~m}^{-3}\left(0.85 \mathrm{~ms}\right.$ after switch-on) to $2.4 \times 10^{19} \mathrm{~m}^{-3}$ (5.35 ms after switch on). The filament quantifier has been determined for these single profiles. The $1 / n_{\mathrm{e}}^{1.5}$ dependence found in section 5.3 has been used to correct the value for $\Theta_{T_{\mathrm{e}}}$ for the central density decrease.

Figure 8 shows the development of the filament amplitude in the ECH switch-on phase. Each point in the figure represents a single TS observation. Therefore the indicated error bars are large. At $t=0 \mathrm{~ms}$ the amplitude of $\Theta_{T_{\mathrm{e}}}$ for a typical ohmic plasma is taken, which is $\Theta_{T_{\mathrm{e}}}=$ $100 \pm 50 \mathrm{eV}$. The saturation level at $550 \mathrm{eV}$ is obtained from the series at $q_{\mathrm{a}}=4.5$ in figure 5 . 

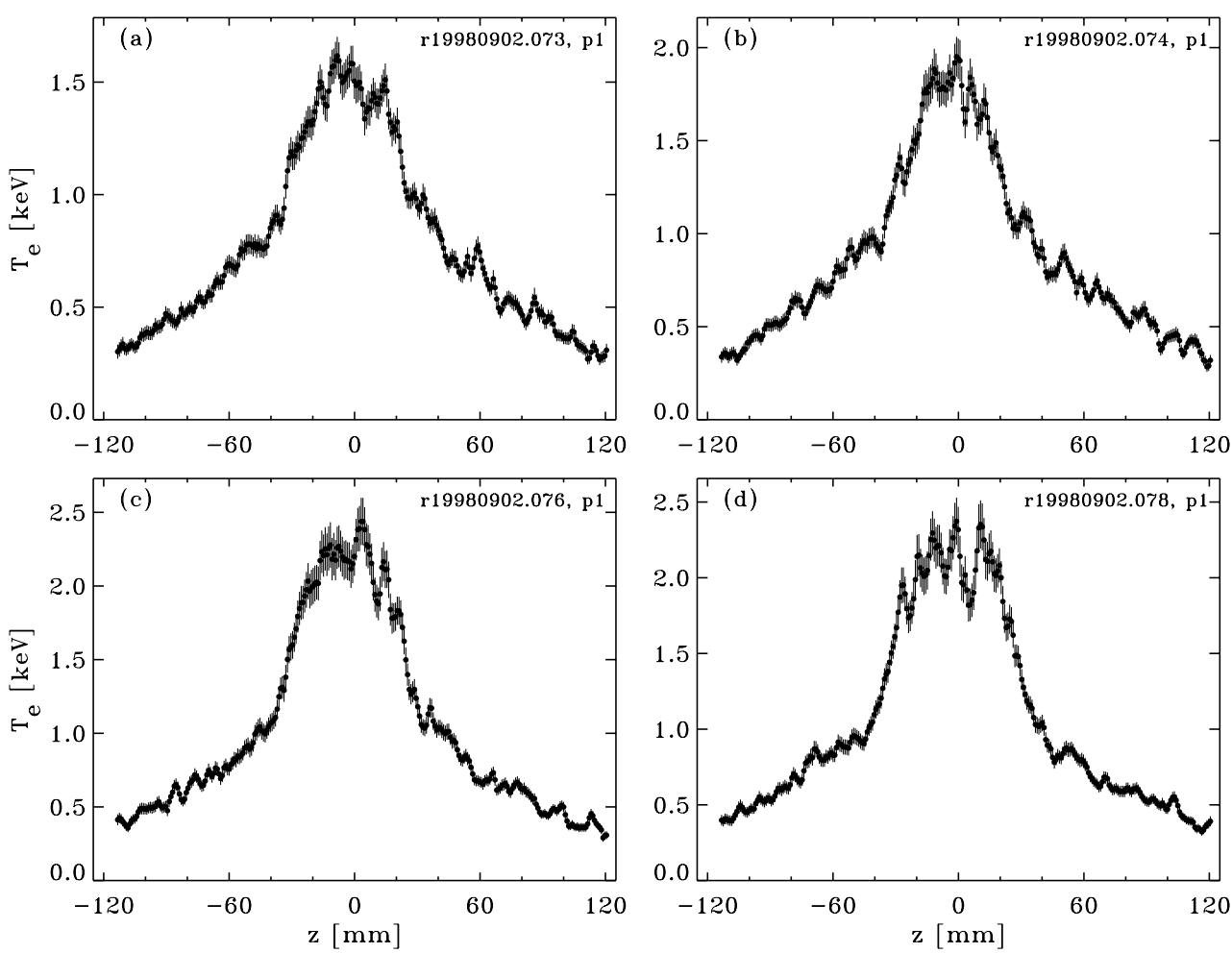

Figure 7. $T_{\mathrm{e}}$ profiles at different timing after ECH switch-on: (a) $0.85 \mathrm{~ms}$, (b) $1.85 \mathrm{~ms}$, (c) $3.85 \mathrm{~ms}$, and (d) $5.35 \mathrm{~ms}$. The central filament amplitude increases. The discharges presented have $q_{\mathrm{a}} \approx 4.5$.

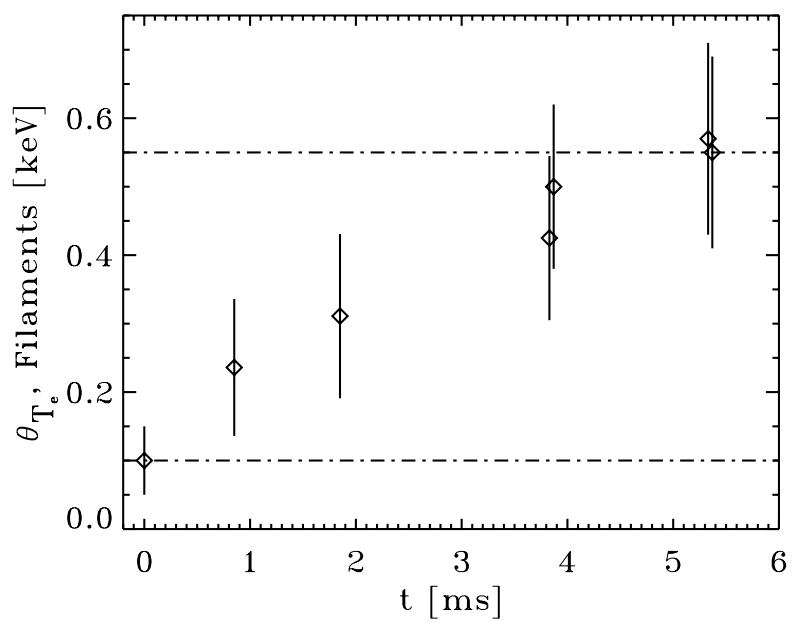

Figure 8. Filament amplitude expressed in $\Theta_{T_{\mathrm{e}}}$ as a function of time after ECH switch-on. In the figure, $\Theta_{T_{\mathrm{e}}}$ for the ohmic plasma is included. The threshold at $550 \mathrm{eV}$ is obtained from the averaged $\Theta_{T_{\mathrm{e}}}$ at $q_{\mathrm{a}}=4.5$ in figure 5. Note that the data are obtained from single TS measurements. The spread is determined by multiplying the spread in figure 5 by the square root of the number of discharges used in that figure. The trend is clear: it takes milliseconds before the maximum filament amplitude is reached. 
Although the error bar on $\Theta_{T_{\mathrm{e}}}$ is large for an individual shot, the measurements clearly show that the filaments develop gradually, taking $5 \mathrm{~ms}$ to reach the saturated amplitude. The associated time constant is estimated at $3 \mathrm{~ms}$. The time constant of the central $T_{\mathrm{e}}$ rise is $1.5 \mathrm{~ms}$.

If the filaments were pre-existing structures that only needed to be heated by the $\mathrm{ECH}$, they should reach their full amplitude within several hundreds of microseconds. Indeed, as is shown in section 8 , when filaments have disappeared in a sawtooth crash, it takes only a few hundred microseconds for them to reappear.

Thus, the time constant for filament creation after ECH switch-on should be interpreted as the time needed to create the required conditions for filamentation. The fact that it is longer than the core energy confinement time suggests that current diffusion, i.e. the modification of the $q$-profile, plays a role in this process.

Filament-like structures have been observed in the ohmic phase of the plasma, as will be presented in section 7.2. However, the difference in creation time of filaments after switch-on of ECH (i.e. $3 \mathrm{~ms}$ ) and the re-occurrence after a sawtooth collapse (a few hundred microseconds) is an indication that the ECH filaments are not the same structures as those observed in ohmic plasmas.

\section{Do filaments only occur in plasmas with central ECH deposition?}

All filaments presented so far were observed in plasmas with central ECH. This section shows a range of experiments where filament-like structures are observed in other conditions. It is meant to show the variety of occurrence of filament-like structures, but does not contain enough data to perform a comparison of the nature of filaments in centrally heated discharges and the structures presented in this section.

We first show the occurrence of filaments in off-axis heated discharges. Then we examine the $T_{\mathrm{e}}$ profiles of normal ohmic discharges. Finally, we report observations of filaments in ohmic discharges which show a transient rise of the central temperature in response to edge cooling (the 'non-local' effect).

\subsection{Do filaments occur at $q>1$ ?}

So far all examples of filaments were found in the central part of the plasma, inside the sawtooth inversion radius, where $q$ is close to one. These plasmas were centrally heated by means of ECH. There is a much smaller set of observations of filaments in plasmas with inverted $q$ profiles, which in RTP are obtained — steady state-by application of off-axis ECH $[19,20,21]$. Figure 9 gives an example of an extreme case, where the ECH resonance was at $\rho=0.6$ (corresponding to $z=100 \mathrm{~mm}$ ). In this case the $T_{\mathrm{e}}$ profile as well as the $p_{\mathrm{e}}$ profile become hollow, while the $n_{\mathrm{e}}$ profile remains peaked.

Inside the hot region, spatial variations of $T_{\mathrm{e}}$ are observed that are very similar to the filaments observed in centrally heated plasmas. Although the amplitude of the off-axis filaments is much smaller than that of the central filaments, their statistical significance is similar, thanks to the small error bars at the lower $T_{\mathrm{e}}$.

Figure 9(d) shows the $q$ profile, calculated on the basis of the measured $T_{\mathrm{e}}$ profile assuming neo-classical resistivity. The value of $q$ in the low shear region is approximately $q=5.5-6$, but the calculation of the $q$ profile is not sufficiently accurate to determine whether the filaments are a resonant phenomenon or not.

In conclusion, there is a limited set of observations of filaments in $T_{\mathrm{e}}$ profiles with a pronounced off-axis maximum, obtained by application of off-axis ECH. In those cases, the value of $q$ is certainly well above unity. 

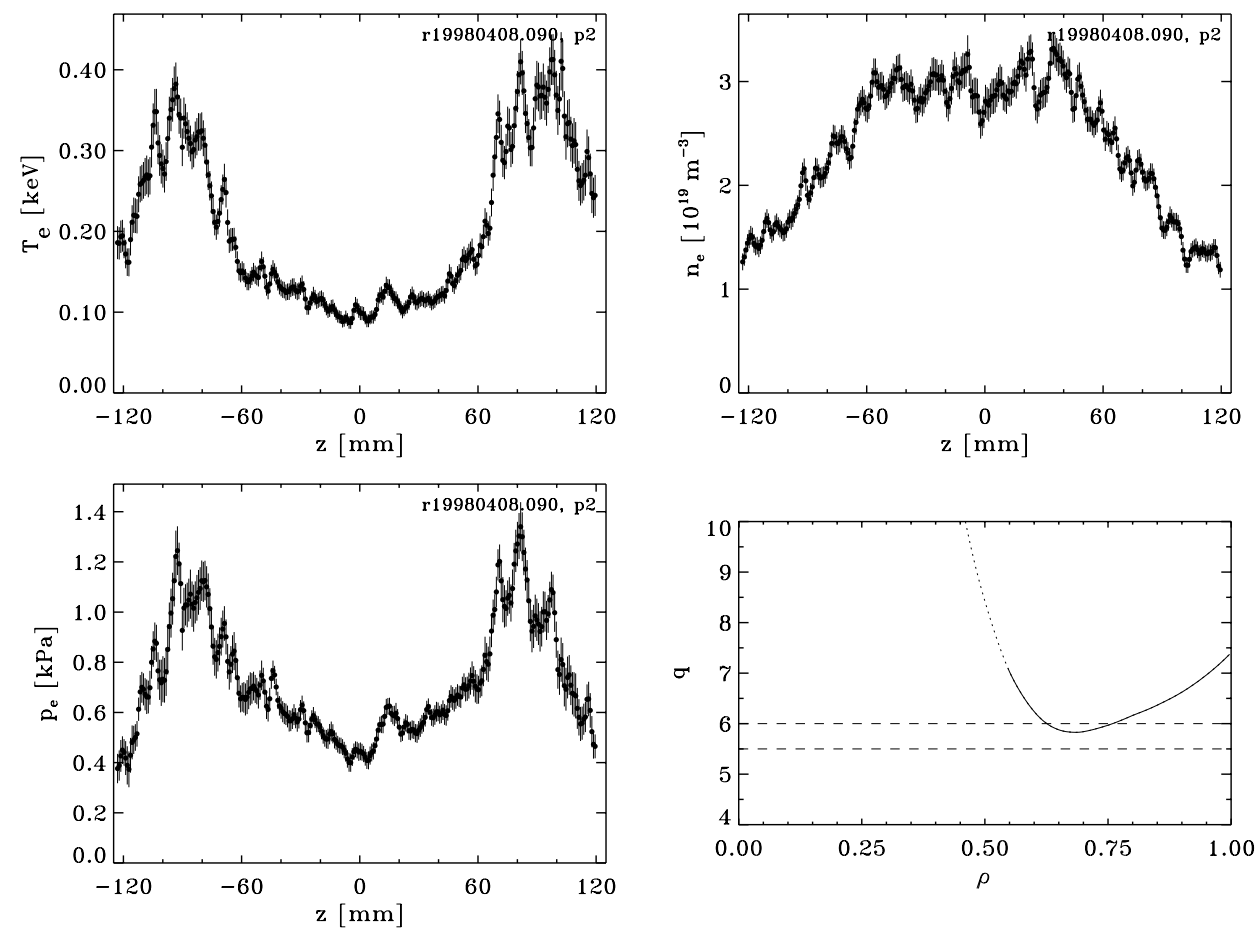

Figure 9. Example of filaments in an off-axis heated plasma. The ECH deposition region is at the $T_{\mathrm{e}}$ maximum. $q_{\min }>5.5 \mathrm{in}$ this plasma, $I_{\mathrm{p}}=60 \mathrm{kA}$, and $B_{\phi} \approx 2.25 \mathrm{~T}$.

\subsection{Do filaments occur in normal ohmic discharges?}

Figure 10 shows $T_{\mathrm{e}}$ profiles of ohmic discharges with $q_{\mathrm{a}}=5$, at three different densities. All profiles show a rich fine structure. Since the error bars are small for this $T_{\mathrm{e}}$ range, especially for the higher densities, these structures are statistically significant. The typical size is at $5-10 \mathrm{~mm}$ similar to that of filaments. The typical amplitude of the structures is $100 \mathrm{eV}$, approximately constant over the profile. There is no clear density dependence. Therefore, despite the fact that the size and relative amplitude of the structures in ohmic discharges are comparable to those of filaments, the phenomenology is rather different. The interpretation of these structures is therefore outside the scope of the present paper. However, it may be clear that if these structures are the fingerprint of a broken magnetic topology, this is significant for the understanding of electron heat transport in tokamaks.

\subsection{Filaments during 'non-local' heating}

In many tokamaks it has been observed that an induced fast $T_{\mathrm{e}}$ drop in the outer layer of the plasma leads to a temporary rise in the central $T_{\mathrm{e}}[22-25,27]$. This phenomenon is often referred to as the 'non-local' effect. In RTP many experiments have been dedicated to this phenomenon, using oblique pellet injection (the pellet passes the plasma centre at $>65 \%$ of the minor radius) to induce the fast edge cooling, and techniques such as modulated ECH to probe the transport.

At $2.5<n_{\mathrm{e}}<3 \times 10^{19} \mathrm{~m}^{-3}, T_{\mathrm{e}}$ filaments have been observed during the transient $T_{\mathrm{e}}$ rise. Figure 11 shows two examples. In those plasmas, no ECH was applied at all. The filament 

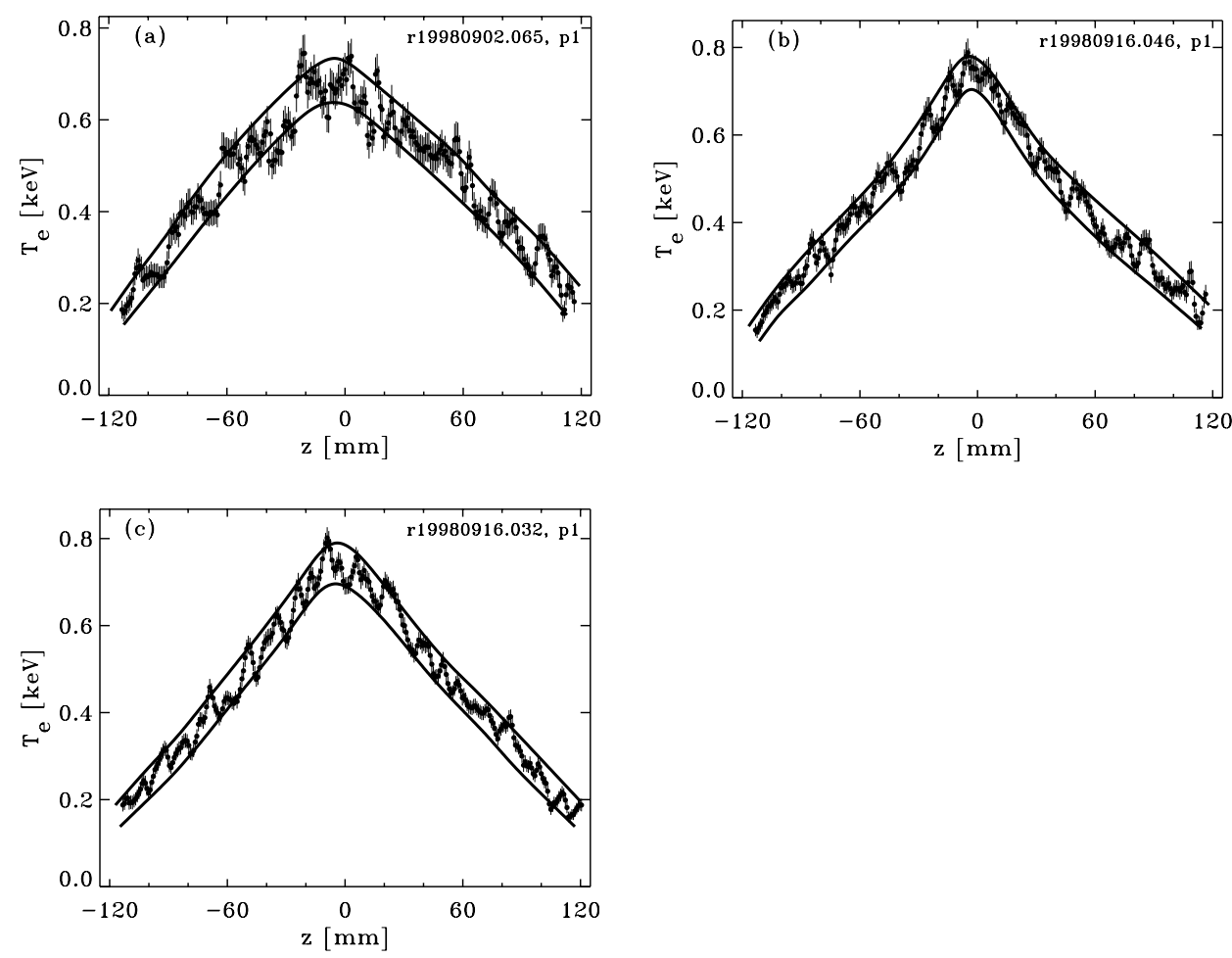

Figure 10. $T_{\mathrm{e}}$ profiles of ohmic discharges with $q_{\mathrm{a}} \approx 5$. The central $n_{\mathrm{e}}(0)$ is (a) 2.7, (b) 4.8 and (c) $6.4 \times 10^{19} \mathrm{~m}^{-3}$. The drawn lines indicate the maximum structure amplitude in the profiles. The upper and lower profiles drawn in each figure are almost parallel, showing that the structures have a constant amplitude throughout the profile.

amplitude is, at 200-300 eV, somewhat smaller than in ECH plasmas, but significantly larger than that of the structures in normal ohmic plasmas.

\section{Filaments and sawtooth activity}

The double pulse feature of the TS diagnostic has been applied to measure $T_{\mathrm{e}}$ profiles just before and just after a sawtooth collapse in the same discharge. Both in the case of non-locally heated plasmas and ECH plasmas the filaments are destroyed by the sawtooth collapse.

Figure 11 shows two examples of non-locally heated discharges. The $T_{\mathrm{e}}$ rise in the plasma centre can be seen in the central ECE measurements. The maximum $T_{\mathrm{e}}$ is reached a few milliseconds after the pellet is injected. With the rise of the central $T_{\mathrm{e}}$, the sawtooth activity is also enhanced. For both discharges the timing of the double pulse TS measurements is indicated in the ECE trace. Example (a) has the first pulse just after a sawtooth collapse. It shows a flat profile without filaments. The second pulse, $400 \mu$ s later, and just before the next collapse, shows three to four filaments. Example (b) shows the reverse case. The first TS measurement is just before a sawtooth crash, the second just after. The $T_{\mathrm{e}}$ profile of the first pulse is filamented while the second is flat.

Thus, filaments observed during the non-locally induced $T_{\mathrm{e}}$ rise, are destroyed by a sawtooth crash. In ECH plasmas sawteeth also have a destructive effect on filaments. 

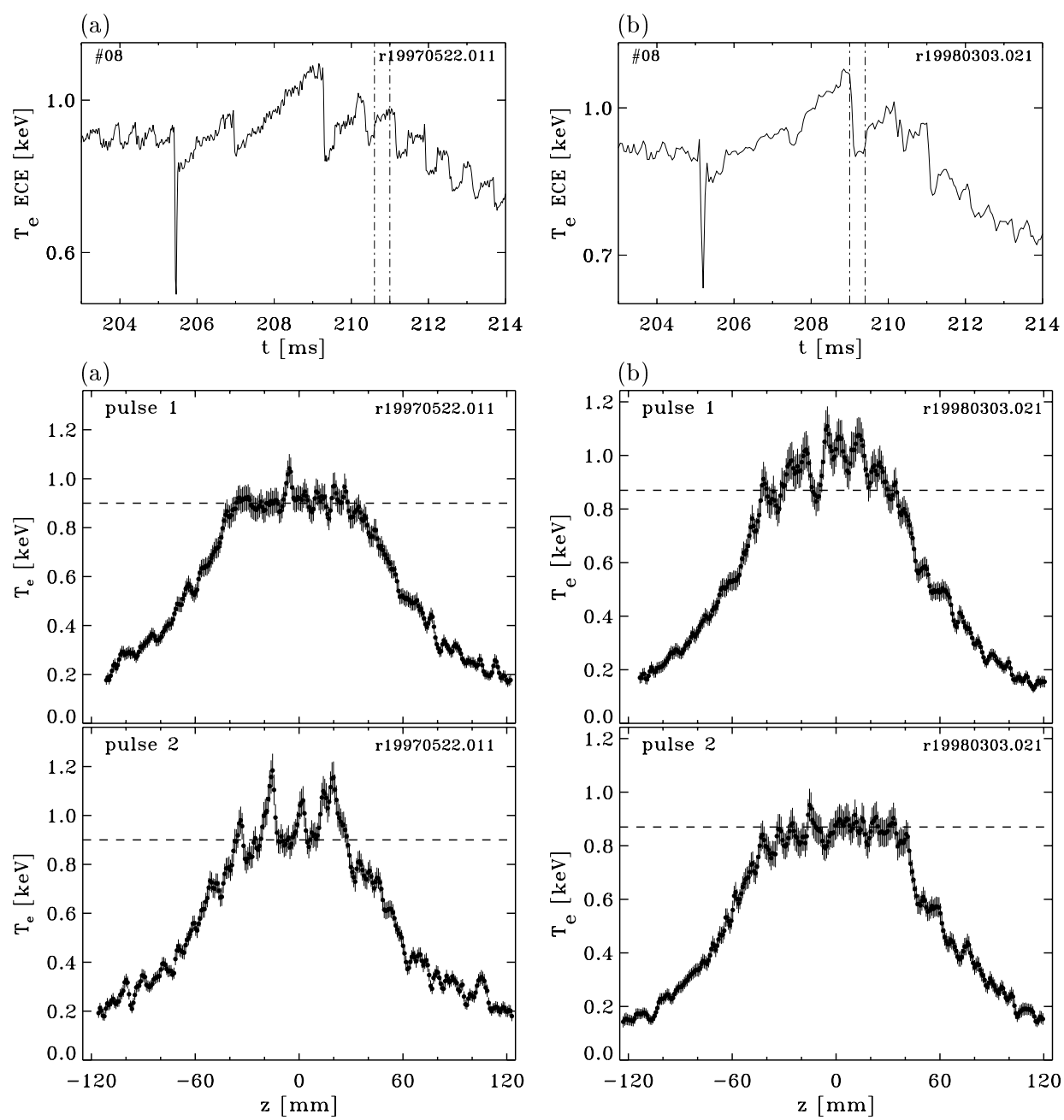

Figure 11. Two examples of filaments in 'non-locally' heated plasmas. The central ECE channels show that $T_{\mathrm{e}}$ in the centre of the plasma rises after the pellet has been injected. The moment of pellet injection is at (a) $205.4 \mathrm{~ms}$ and (b) $205.2 \mathrm{~ms}$ at the sudden dip in the ECE signal. Sawteeth have a destructive effect on the filaments in these plasmas. In (a) the first pulse is just after a sawtooth collapse and shows a flat profile. The second profile, $400 \mu$ s later, shows filamentation of the core. In (b) the first pulse is just before a crash and is filamented, while the second profile just after the crash is flat.

Figure 12 shows a central ECE trace of a discharge with $q_{\mathrm{a}}=5.8\left(I_{\mathrm{p}}=60 \mathrm{kA}\right)$ and $n_{\mathrm{e}}(0)=1.5 \times 10^{19} \mathrm{~m}^{-3}$. Central ECH is launched under an angle of about $+20^{\circ}$ with respect to the toroidal field $B_{\phi}$, driving a non-inductive current of $\sim 10 \mathrm{kA}$. This peaks up the current density profile and enhances the sawtooth activity. In three well reproducing discharges, single-pulse TS is fired in different stages of the sawtooth cycle, as indicated in figure 12. The figure also shows the three $T_{\mathrm{e}}$ profiles. The profile just before the sawtooth crash shows central filaments with an amplitude of 500-700 eV. During the crash a $T_{\mathrm{e}}$ profile is measured which shows the spectacular displacement of the hot core. The profile after the crash is flatter, and the amplitude of the structures in the centre is lower than those in the profile before the crash. 

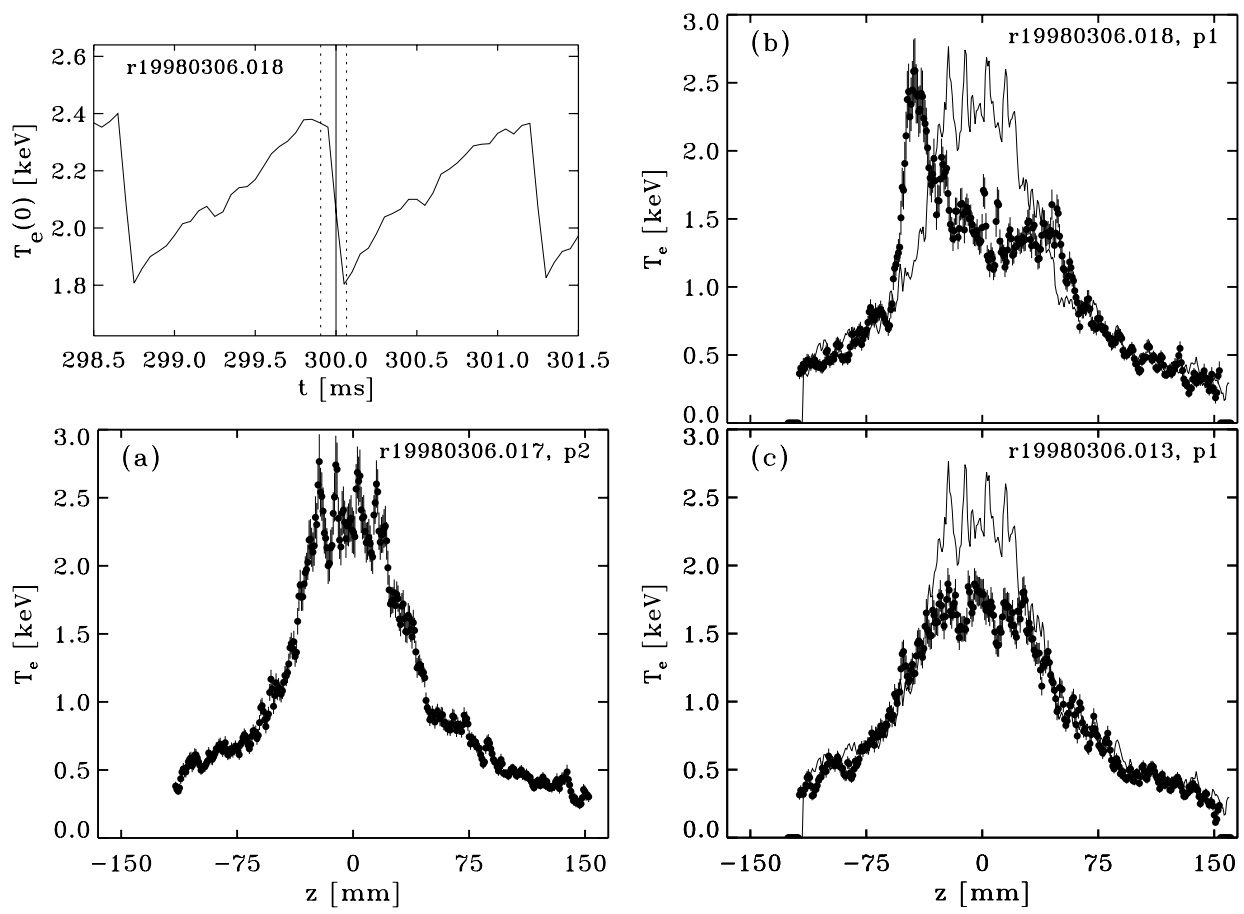

Figure 12. TS profiles in different stages of a sawtooth cycle in well reproducing plasmas with ECCD. The timing of the pulses in relation to the sawtooth phase is indicated in the central ECE time trace (top left). (a) Just before the sawtooth crash, with high central $T_{\mathrm{e}}$ and a filamented core. (b) Profile inside the sawtooth crash, showing the displacement of the hot core. (c) After the sawtooth crash, showing the flattened top of the profile. The profile of (a) is overplotted in (b) and (c).

Outside the inversion radius the profiles before and after the crash are very similar.

Figure 13 shows two examples of double pulse measurements in a sawtoothing plasma. The time resolution of the ECE in this experiment is higher than in the examples of figures 11 and $12(6 \mu$ s instead of $50 \mu \mathrm{s})$. The central ECE trace shows a large $m / n=1 / 1$ precursor. The first example shows the footprint of the large odd mode in the first profile. The high $T_{\mathrm{e}}$ region is filamented. The second profile is after a sawtooth collapse and shows no filamentation. The second example shows the reverse case. The first profile is just after a collapse and is flat. The second profile is filamented again.

In section 6 it was shown that it takes $\sim 3 \mathrm{~ms}$ for the filaments to develop after ECH is switched on. This long time scale was interpreted as the time needed to achieve the conditions in which the filaments can grow. In the case of the sawtooth crash, the conditions were already favourable for filamentation before the crash, and this is still the case after the crash. Therefore, the few hundred microseconds within which the filaments reappear after a sawtooth crash may be interpreted as the energy confinement time of a filament. This is consistent with the observation that filaments persist for a few hundred microseconds after ECH is switched off. 

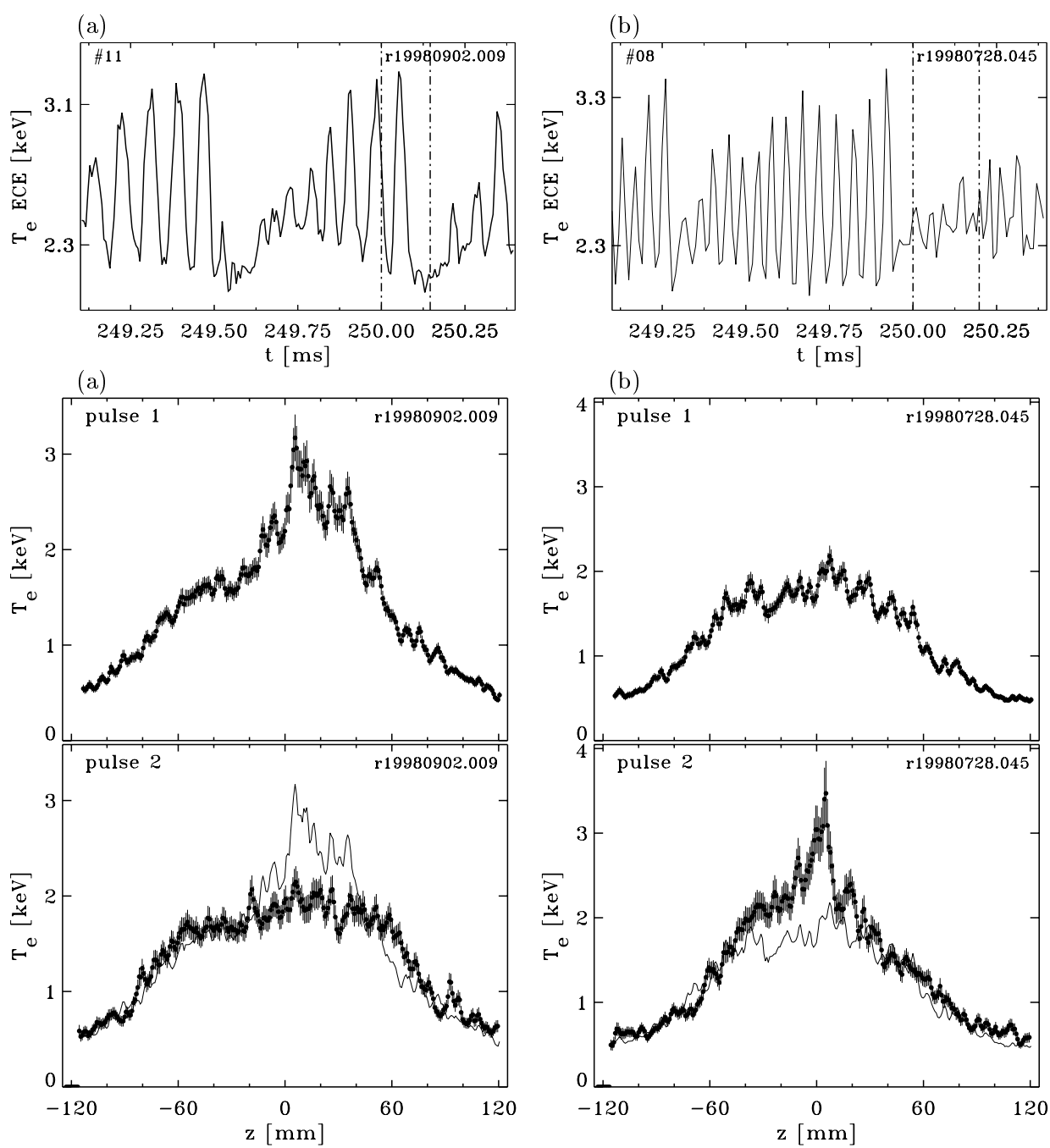

Figure 13. Two examples of the effect of sawteeth on filaments. Both show a large $m / n=1 / 1$ sawtooth precursor on the central ECE channel. In (a) the double pulse TS measurements are just before and just after the crash, as indicated by the vertical lines in the ECE time trace. The profile before the crash shows the footprint of the $m / n=1 / 1$ mode and filaments. The second profile is flattened. In (b) both pulses are after the crash. The first profile is flat, and the second $200 \mu$ s later is filamented again. (a) $q_{\mathrm{a}}=3.1, n_{\mathrm{e}}(0)=2.7 \times 10^{19} \mathrm{~m}^{-3}$, (b) $q_{\mathrm{a}}=4.6, n_{\mathrm{e}}(0)=2.3 \times 10^{19} \mathrm{~m}^{-3}$.

\section{Topology of filaments}

As was concluded in section 4, the filaments can only be understood if they are closed structures. In the following, therefore, only such toroidally closed structures will be considered. In this section interpretations for filamented plasmas will be compared to a series of experiments. First the interpretations will be introduced and then the experiments will be presented. Finally an attempt is made to link the interpretations to the experiments.

In $[12,13]$ it is assumed that the filaments are separate flux tubes closing back on themselves. These tubes could occur independently of the presence of a larger $m=1$ sawtooth 
precursor. They would have higher confinement than the ambient plasma and therefore their $T_{\mathrm{e}}$ is higher. Because of this higher $T_{\mathrm{e}}$ the conductivity inside the filament is increased and the current density is enhanced. The filaments therefore could be interpreted as positive magnetic islands.

Mirnov [15] suggested a nonlinear scenario for positive magnetic islands in a tokamak plasma. These islands carry a higher current density than the ambient plasma. A necessary condition for these positive islands is low magnetic shear. The formation of flux tubes around current concentrations in low magnetic shear has also been studied in [28].

Studies of electron MHD by Kuvshinov et al show that under strong, local heating dipole vortices form of which one part absorbs the heat and remains as a hot filament [29, 30]. While these are preliminary results, they do show how local electron heating can lead to formation of structures in the plasma.

These schemes all result in filaments as toroidal tubes closing back upon themselves.

In [14] a pseudo-tomography of the filamented central region of the poloidal cross section is made using the time resolved ECE measurements and the rotation of the plasma. The tomography shows that what appear as filaments in a one-dimensional $T_{\mathrm{e}}$ profile, form a single $m=n=1$ island with a very complex shape. This island, and thus the filaments, are then equated to the sawtooth precursor.

Inspired by these observations Porcelli et al have constructed a model which considers a growing $m=1$ sawtooth precursor in rotating plasma with a heat source which is essentially narrower than the island width [16]. Figure 14 shows a few results obtained from the model. In figure 14(b) and (c) two examples are given of the $T_{\mathrm{e}}$ profile in a poloidal cross section. Clearly, the $m=1$ island structure can be distinguished. Furthermore, ring-shaped regions with sharp $T_{\mathrm{e}}$ gradients have developed. Figures 14(d)-(f) show examples of $T_{\mathrm{e}}$ profiles along a chord. Typically four to five peaks show up in the profiles.

Figure 15 shows schematic drawings of the two options, filaments as part of a deformed $m=1$ sawtooth precursor or filaments as toroidal tubes. The main difference is the poloidal extension of areas with peaked $T_{\mathrm{e}}$.

Three sets of experiments were conducted in RTP to study the topology of filaments: (i) Taking TS profiles at different positions relative to the magnetic axis, to check if the appearance of the filaments depends on their proximity to the $q=1$ surface (section 9.1). (ii) Setting the $\Delta t_{\mathrm{TS}}$ of the double pulse TS to the rotation period of the filaments to investigate their reproduction after a full rotation (section 9.2). (iii) Relating the filament amplitude to the amplitude of a sawtooth precursor present in the plasma. This way the correlation between the two can be investigated (section 9.3).

\subsection{TS profiles: scan of the major radius}

The TS laser is aligned to the geometrical centre of the poloidal cross section of the torus, which normally is the centre of the last closed flux surface. With a Shafranov shift of $20 \mathrm{~mm}$, the TS profile misses the magnetic axis of the plasma. However, the plasma can be shifted radially by up to $\Delta R= \pm 20 \mathrm{~mm}$ in the equatorial plane. In a series of discharges, $\Delta R$ was varied between -17 and $+13 \mathrm{~mm}$. All discharges were centrally heated with $\mathrm{ECH}$, and had $q_{\mathrm{a}}=4.6$. Unfortunately, the line integrated density varied between 4 and $6 \times 10^{18} \mathrm{~m}^{-2}$ in this series. There is a strong dependence of the filamentation amplitude on the density (see section 5.3) for which we did not correct.

In figure $16 \operatorname{six} T_{\mathrm{e}}$ profiles from this series are shown. First we note that the steep gradient, which marks the position of the $q=1$ radius [19], is largest for $\Delta R=-17 \mathrm{~mm}$, while for $\Delta R=+13 \mathrm{~mm}$ the hot core is only barely touched or perhaps missed altogether. This is in 

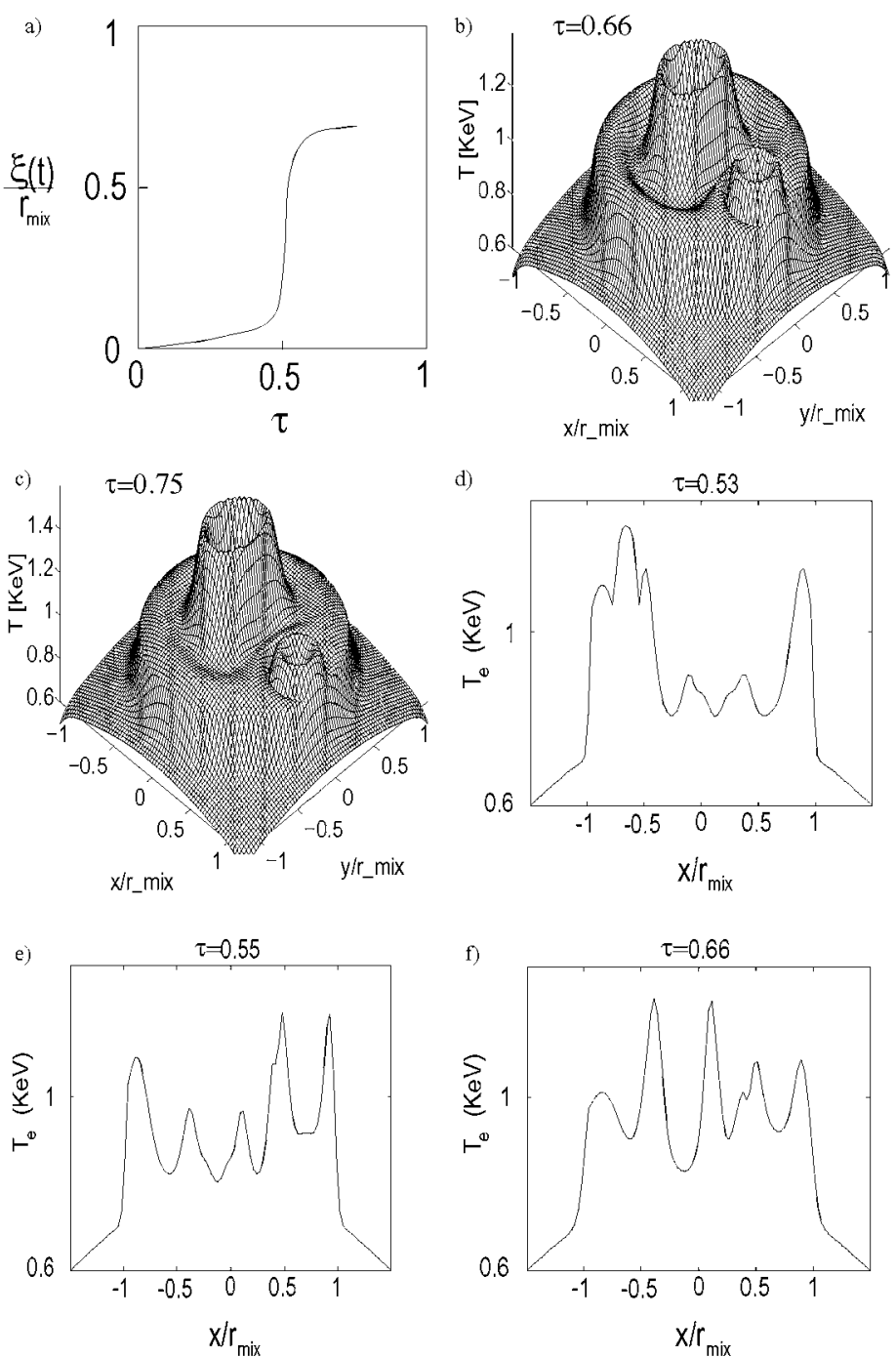

Figure 14. Results from the model by Porcelli et al. (a) Displacement of the magnetic axis $\xi$ normalized to the sawtooth mixing radius $r_{\text {mix }}$ as a function of the normalized sawtooth period $\tau=t / \tau_{\text {saw }}$. (b) and (c) Examples of the three-dimensional reconstruction of $T_{\mathrm{e}}$. The figures show the $m=1$ sawtooth precursor, in which sharp $T_{\mathrm{e}}$ gradients have developed. (d)-(f) Examples of line cross sections of $T_{\mathrm{e}}$ at different time points. (From [16], with kind permission of the author.)

agreement with the estimated Shafranov shift of $20 \mathrm{~mm}$. The appearance of the filaments does not depend on the cross section. There are always multiple peaks, which are distributed rather randomly over the hot core. Especially cuts close to the edge of the hot region, i.e. figure 16(d) and (e), appear to be incompatible with the model by Porcelli.

\subsection{Appearance of filaments under rotation}

The double pulse feature of the high resolution TS diagnostics is used to study the rotation of filaments. For this we have to assume that the lifetime of filaments is longer than their rotation period. Based on their persistence after $\mathrm{ECH}$ is switched off, the life time of filaments in RTP 

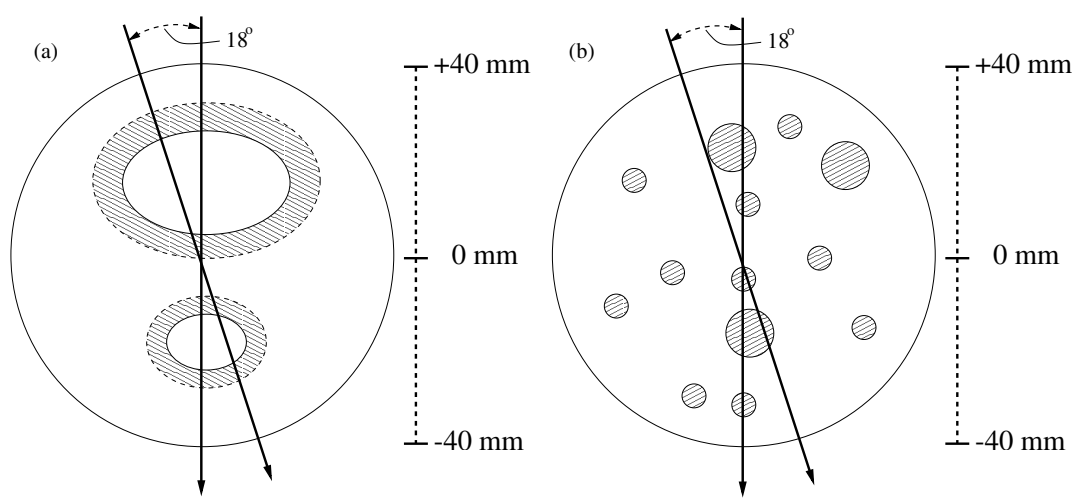

Figure 15. Schematic drawing of the two options for a filamented plasma centre (see text). The dashed area indicates higher $T_{\mathrm{e}}$ than outside. The outer circle indicates the sawtooth mixing radius $r_{\text {mix }}$, indicating the $q=1$ surface. The dimensions are adapted to RTP dimensions: filaments of $5-10 \mathrm{~mm}$ width on a vertical chord and in a central region of $\approx 8 \mathrm{~cm}$ diameter. (a) Rings with higher $T_{\mathrm{e}}$ according to Porcelli, figure 14. (b) Tube-like filaments of 5 and $10 \mathrm{~mm}$ diameter. The main difference between the two options lies in the poloidal extension of the high $T_{\mathrm{e}}$ area. The two arrows drawn indicate a $5 \%$ mismatch between the set $\Delta t_{\mathrm{TS}}$ of the double pulse TS and the rotation of the 'filaments' (see section 9.2).

Table 1. Reproduction of $m=1$ mode and filaments at $\Delta t_{\mathrm{TS}}=90 \mu \mathrm{s}$ in 100 similar discharges. All discharges feature filamented $T_{\mathrm{e}}$ profiles.

\begin{tabular}{llll}
\hline $\begin{array}{l}m=1 \text { mode } \\
\text { present }\end{array}$ & $\begin{array}{l}\text { Number of } \\
\text { shots }\end{array}$ & $\begin{array}{l}\text { Number of } \\
\text { reproducing } \\
m=1 \text { mode }\end{array}$ & $\begin{array}{l}\text { Number of } \\
\text { reproducing } \\
\text { filaments }\end{array}$ \\
\hline Yes & 54 & 50 & 2 \\
No & 46 & - & 4 \\
\hline
\end{tabular}

was estimated at several hundreds of microseconds [12], i.e. much longer than a single plasma rotation.

For this experiment we want to set the separation time $\Delta t_{\mathrm{TS}}$ of the double pulse TS to a single rotation of a filament. Unfortunately, single filaments are too small to be detected by time resolved measurements, like ECE or magnetics. Therefore, we set $\Delta t_{\mathrm{TS}}$ to a typical rotation time of an MHD mode. Figure 17 shows a large rotating $m=n=1$ precursor in a centrally heated plasma with $\tau_{\mathrm{MHD}}=90 \mu \mathrm{s}$. The subsequent $T_{\mathrm{e}}$ profiles with $\Delta t_{\mathrm{TS}}=\tau_{\mathrm{MHD}}$ are shown in figure 18(a). Both $T_{\mathrm{e}}$ profiles clearly show the presence of the odd mode, and the very peaked shifted hot core.

One hundred similar discharges were produced with favourable conditions for filaments, i.e. central ECH and low density. Table 1 shows the results of reproduction for these discharges. Only six out of 100 discharges featured reproducing filaments. Figure 18(b) shows an example where no $m=n=1$ mode has been observed, but where the filaments in two subsequent profiles reproduce.

In the 54 discharges with a visible $m=n=1$ mode, $\tau_{\text {MHD }}$ varied from 80 to $100 \mu$ s. Still most of them show a reproducing $m=n=1$ mode in two subsequent TS profiles. This is not surprising because of the typical large poloidal extension of such an MHD mode.

Regarding the reproduction of the filaments the following statements can be made:

- Filaments can be reproduced by setting $\Delta t_{\mathrm{TS}}$ to $\tau_{\mathrm{MHD}}$. Thus the filaments have a similar 

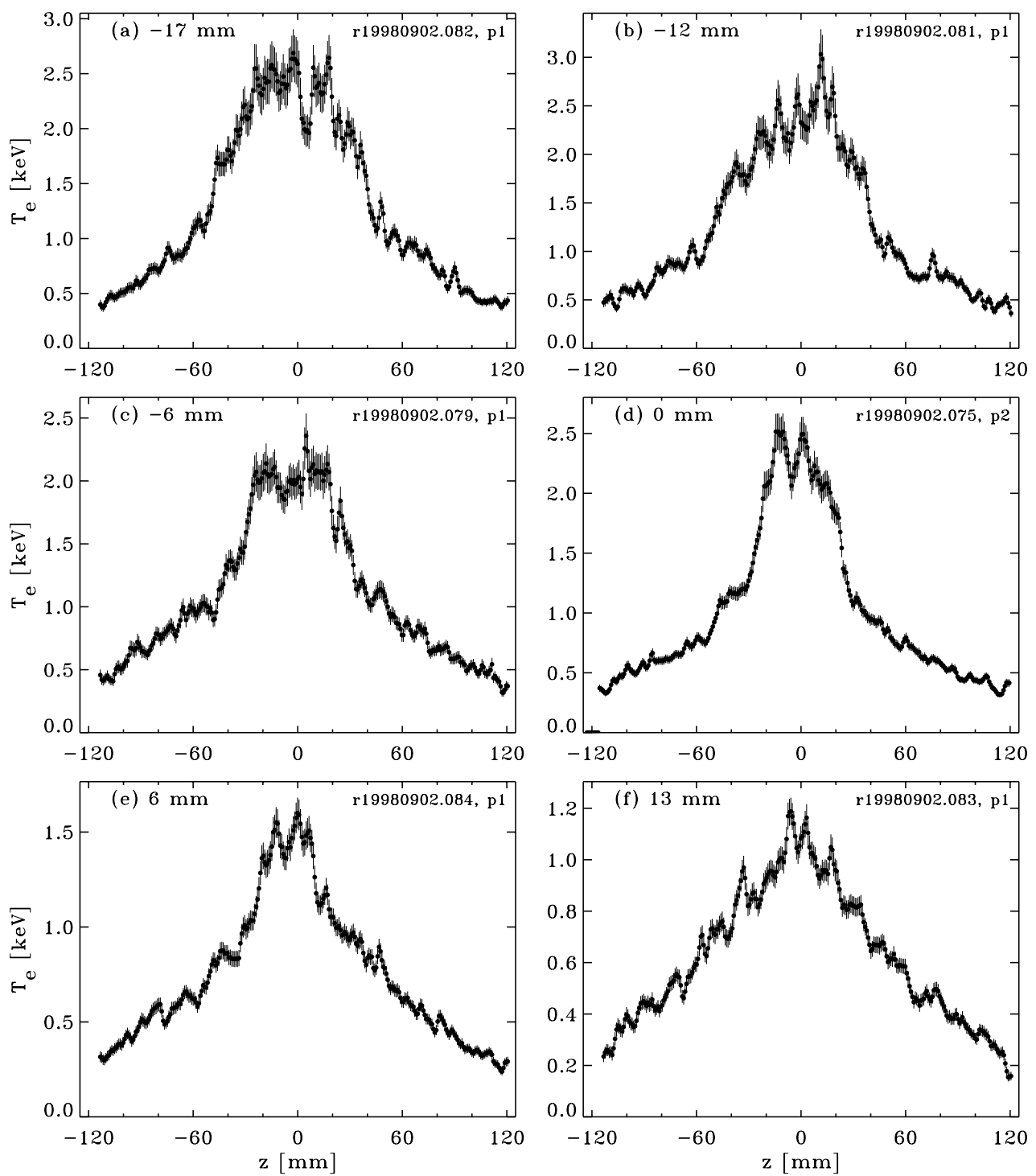

Figure 16. Measured $T_{\mathrm{e}}$ profiles of plasmas that were horizontally shifted with $\Delta R=(\mathrm{a})-17 \mathrm{~mm}$, (b) $-12 \mathrm{~mm}$, (c) $-6 \mathrm{~mm}$, (d) $0 \mathrm{~mm}$, (e) $6 \mathrm{~mm}$, and (f) $13 \mathrm{~mm}$. All profiles show single peaks. The profiles (a)-(d) have a line integrated density of 4-4.5 $\times 10^{18} \mathrm{~m}^{-2}$, while for (e) and (f) the line integrated density is $\sim 6 \times 10^{18} \mathrm{~m}^{-2}$.

rotation period to the large mode.

- Reproduction of filaments is very difficult. Only six out of 100 discharges showed reproducing filaments, thus indicating that their poloidal extension is small.

The first statement suggests that the rotation of filaments is coupled to the rotation of the $m=n=1$ mode. However, there is no reason why independent modes could not rotate with a similar rotation frequency. So no exclusive answer can be given on the correlation between the $m=n=1$ precursor and filaments. 


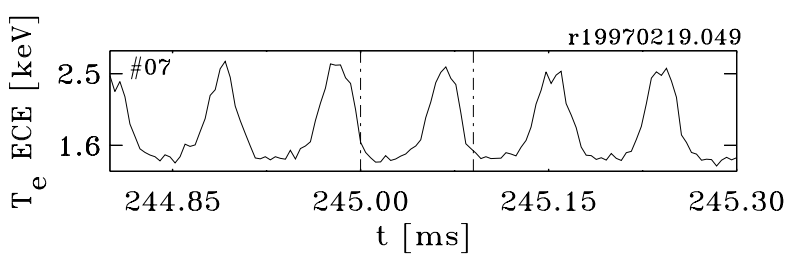

Figure 17. Time trace of a central ECE channel showing the rotation of a large $m=n=1$ mode. The timing of the TS is set to $90 \mu \mathrm{s}$, which is approximately one period of rotation.
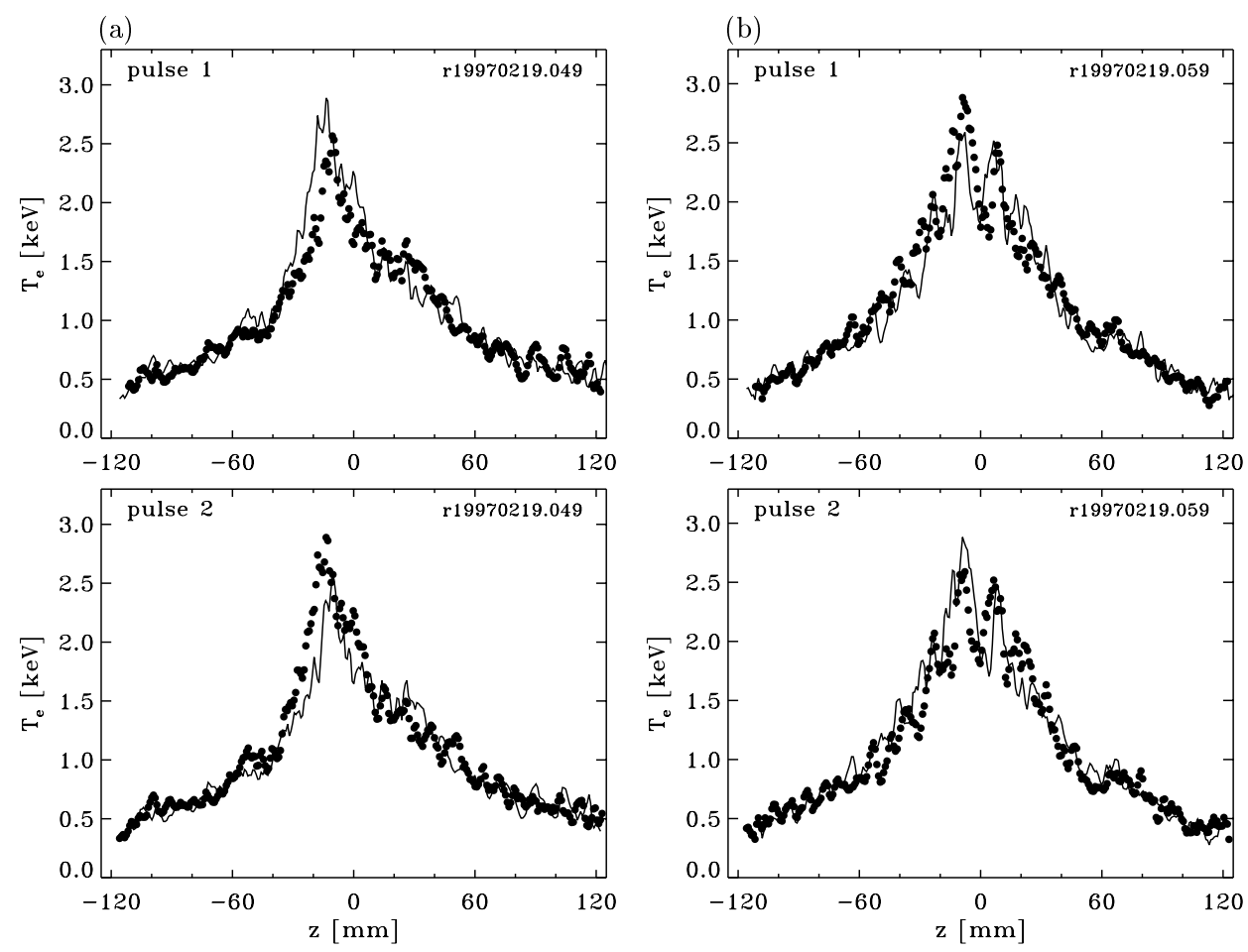

Figure 18. Two examples of double pulse TS measurements with $\Delta t_{\mathrm{TS}}=90 \mu \mathrm{s}$. The first pulse is overplotted in the figure for the second pulse and vice versa. (a) Both profiles show the reproduction of the very peaked hot core in the left wing of the profile and the low $T_{\mathrm{e}}$ area in the right wing. (b) The two filaments near $z=-10$ and $+10 \mathrm{~mm}$ are reproduced. In this case no large mode was present. For both (a) and (b), $n(0)=2.0 \times 10^{19} \mathrm{~m}^{-3}$, and $q_{\mathrm{a}} \approx 6, B_{\phi} \approx 2 \mathrm{~T}$, and $I_{\mathrm{p}}=60 \mathrm{kA}$. The error bars are approximately $150 \mathrm{eV}$ in the centre and are left out for clarity.

Figure 15 simulates the reproduction of filaments. If the filamentary structures have similar poloidal extension as the large $m=n=1$ mode, like in figure 15(a), then filament reproduction should be as successful as the reproduction of the $m=n=1$ mode. This is especially because the structures in figures 14(b) and (c) seem to remain similar for at least one rotation period. On the other hand, if the poloidal extension is limited, like in figure 15(b), it is much harder to reproduce the filaments in two subsequent measurements.

In conclusion, from these experiments we cannot distinguish whether the filaments are coupled to the $m=n=1$ mode. The observations in this section are in favour of filaments as structures with a small poloidal extension. The interpretation of filaments as tube-like 

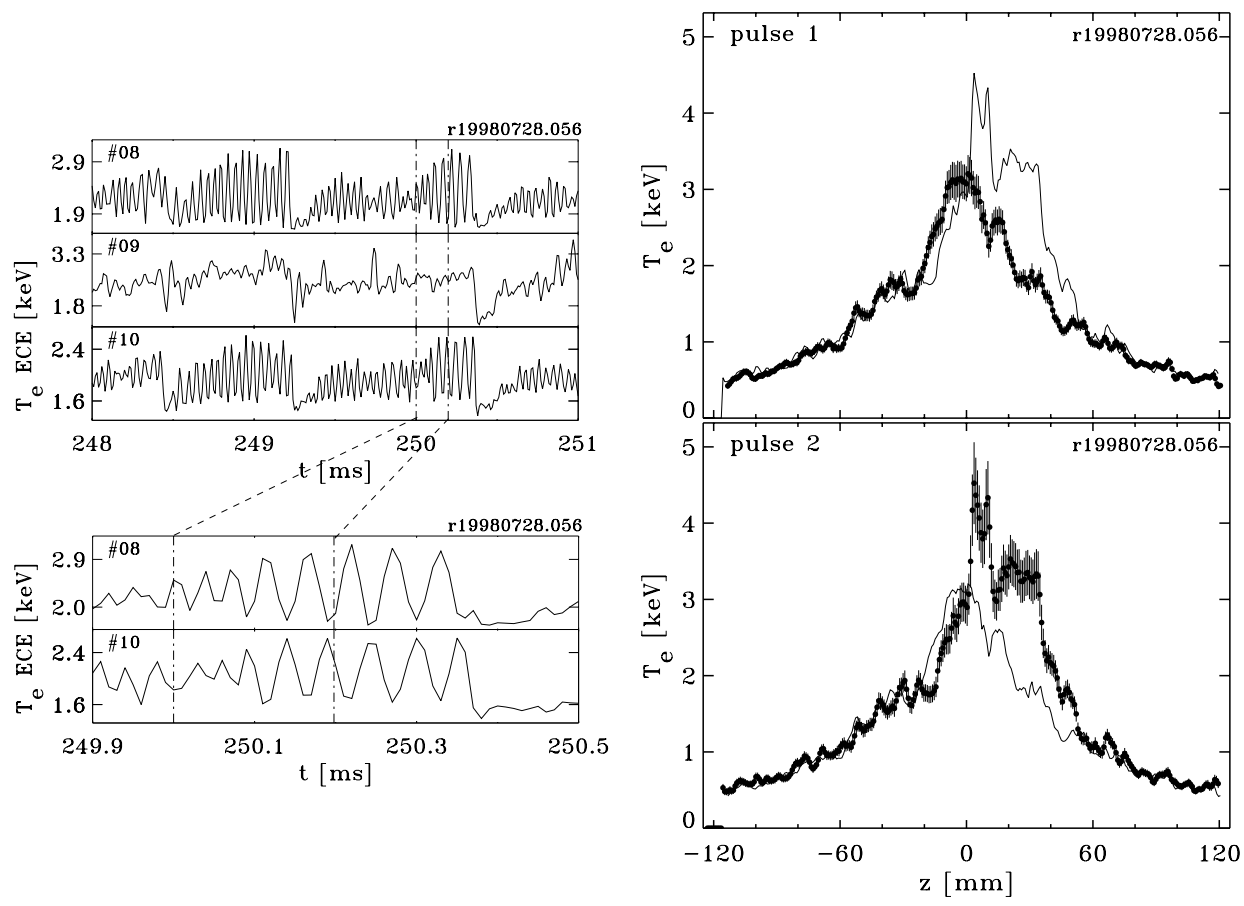

Figure 19. ECE channels showing sawteeth with large $m / n=1 / 1$ precursors. ECE channel \#9 lies on the magnetic axis, channels \#8 and \#10 are in counter phase. The first profile shows the footprint of the $m / n=1 / 1$ island on both sides of the profile. The second pulse shows the footprint on the left side of the profile and a hot filament in the high $T_{\mathrm{e}}$ part of the profile.

structures fits these observations.

\subsection{Filaments and sawtooth precursors}

Figure 19 shows an example of a sawtoothing discharge. The central ECE channels show the $m=1$ character of the mode. The central channel is more or less quiet, and does not show the mode, while the neighbouring channels show the mode in counter phase. A double pulse TS measurement has been performed, and the two pulses both fall in the rising phase of a sawtooth. Both $T_{\mathrm{e}}$ profiles of the TS measurement show the footprint of the odd mode. The first pulse shows flat $T_{\mathrm{e}}$ regions symmetric to the left and to the right of the centre, and the second pulse shows a flat region in the left wing of the profile. A large filament is visible in the profile of the second pulse.

Figure 20 shows a TS measurement in a quiet phase of the sawtooth. The central ECE channels show that in this example sawteeth are less frequent, and moreover, the amplitude of the $m=n=1$ precursor is lower. The TS $T_{\mathrm{e}}$ profile is taken at a time that little mode activity is visible in the ECE measurement. This at least shows that the occurrence of filaments does not require a large $m=1$ sawtooth precursor in the plasma. However, since the spatial resolution of the ECE system is worse than that of the TS system, there still could be a small $m=1$ mode present, undetectable for the ECE system.

Porcelli's model does not contain a quantitative requirement on the amplitude of the $m=1$ sawtooth precursor. This experiment therefore does not exclude his model to describe 
(a)

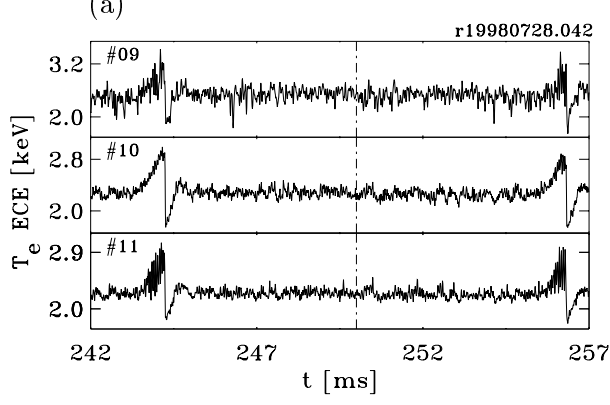

(b)

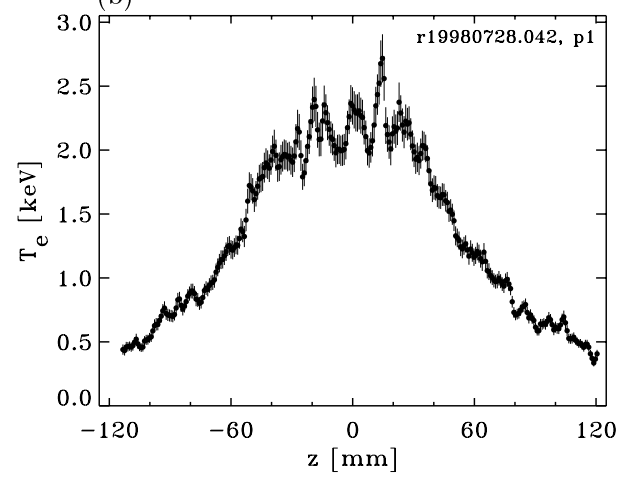

Figure 20. The central ECE channels show that at the time of the TS pulse, no $m / n=1 / 1$ activity is present. Still the TS $T_{\mathrm{e}}$ profile shows filaments. The ECH deposition is just inside the sawtooth inversion radius.

filaments. However, it is clear that the difference in precursor amplitude in figures 19 and 20 is large compared to the relative filament activity in both examples. Therefore, his model needs a study on the dependence of filaments on the amplitude of the sawtooth precursor.

\section{Summary and discussion}

\subsection{Summary of observations and interpretation of topology}

We have presented experimental data showing plasma filamentation in RTP. The filaments are only resolved by the high resolution TS diagnostic, and appear as multiple peaks in the $T_{\mathrm{e}}$ and $p_{\mathrm{e}}$ profiles, while the spatial structures in the $n_{\mathrm{e}}$ profile have a much smaller amplitude.

It was shown that the filaments are not diagnostic artefacts, because (a) their amplitude and spectrum are well above the noise level, and (b) they show a dynamic behaviour that identifies them as plasma physical phenomena: they take several milliseconds to develop after switching on ECH, and they are wiped out in a sawtooth crash. Their reappearance within a few hundred microseconds after a sawtooth crash indicates that their energy confinement is of the order of several hundreds of microseconds. Their lifetime is at least that. The filament amplitude does not strongly depend on $q_{\mathrm{a}}$, but a strong dependence of amplitude on plasma density was found: $\Theta_{T_{\mathrm{e}}}=n_{\mathrm{e}}^{-1.5}$. It was also found that filament-like structures occur in ohmic plasmas. These structures show a different behaviour in terms of density dependence. So far it is not clear how these relate to the filaments in additionally heated plasmas. We know from the duration of filament occurrence after ECH switch-on that the central ECH filaments are not the 'heated' ohmic filaments. Filaments in additionally heated discharges with non-local heating using pellets, and filaments in plasmas with off-axis $\mathrm{ECH}$, are assumed to have a similar nature as those observed in centrally ECH plasmas. However, the database of these observations is not large enough to study, for example, their rotation and their relation to other MHD modes.

The topology of the filaments is a subject that needs further study. In section 9 three experiments have been performed in an attempt to reveal this topology. A scan of the major radius of the plasma covers profiles through the magnetic axis and profiles just touching the $q=1$ surface. All profiles show multiple peaked $T_{\mathrm{e}}$ profiles. A second experiment tries to match the double pulse TS separation time with the rotation time of filaments. It shows that this 
rotation time is similar to that of a large $m=1$ mode in the plasma. In addition, it shows that filaments have a very limited poloidal extension. The third experiment shows that filaments occur independently of the presence of a large $m=1$ sawtooth precursor.

These observations can be compared to the two interpretations of filaments introduced in section 9. First, it must be stated that these interpretations are not comprehensive and that further theoretical work on the modelling of filaments needs to be performed. Comparing the RTP interpretation of filaments as closed tube-like structures with the model of Porcelli et al gives the following topological differences: (i) the RTP model assumes independent MHD structures while the Porcelli filaments are coupled to the presence of a sawtooth precursor; (ii) the RTP filaments are tube-shaped, while the Porcelli filaments are cuts through a poloidally extended shape with a crater-like structure.

The RTP interpretation matches with all experiments performed in section 9. With tubelike filaments it is possible to get multiple peaked $T_{\mathrm{e}}$ profiles in all cross sections (section 9.1). Moreover, the reproduction of tube-like filaments after one rotation is difficult (section 9.2). Finally, the occurrence of tube-like filaments is not limited by the absence of a large $m=1$ sawtooth precursor (section 9.3). The fact that filaments reappear after one rotation period of the $m=n=1$ mode does not exclude that filaments are independent modes.

However, the latter is in favour of Porcelli's model. It may indicate that the peaks in the $T_{\text {e }}$ profile are connected to the $m=n=1$ sawtooth precursor. In Porcelli's model it is also possible to get multiple peaks in the $T_{\mathrm{e}}$ profiles (section 9.1), although it is not possible to have multiple peaks close to the $q=1$ surface, i.e. the sawtooth inversion radius (see figures 14 and 15). Furthermore, the reproduction of poloidally extended filaments after one rotation should be easy, which does not match the results in section 9.2. The experiments in section 9.3 do not exclude Porcelli's model because of lack of spatial resolution of the ECE system. This means that it cannot be excluded that a small $m=1$ precursor is present in the plasma. It is therefore important in the Porcelli model to study the dependence of filament occurrence on sawtooth precursor amplitude.

In conclusion, we can say that the RTP observations, if not exclusive, are favourable for the interpretation of filaments as closed flux tubes rather than part of a complex $m=n=1$ sawtooth prescursor like in Porcelli's model.

\subsection{Physical aspects of tube-like filaments}

Since the experiments are favourable for filaments as tube-like structures we concentrate on this interpretation and discuss some physical aspects and properties of such filaments.

It is interesting to compare the typical width of the filaments with fundamental length scales in the plasma. First, we note the filaments are one to two orders of magnitude larger than the Debije length and the electron Larmor radius. The filaments are only several times larger than the width of electron banana orbits (1-5 mm, depending on the distance to the axis), the ion Larmor radius $(1 \mathrm{~mm})$ and the electron inertial skin depth $(1 \mathrm{~mm})$. Thus, there appear to be no fundamental problems with the size of the filaments, but the observed filaments are close to the smallest size that can be expected on theoretical grounds.

To investigate the power balance in a tube-like filament, first the input power must be estimated. For a typical filament in the centre of an ECH heated plasma, the ohmic power density is estimated at $p_{\Omega} \approx 1 \mathrm{MW} \mathrm{m}^{-3}$ (taking $T_{\mathrm{e}}=2 \mathrm{keV}, j=7 \mathrm{MA} \mathrm{m}^{-2}$, and Spitzer resistivity with $Z_{\text {eff }}=2$ ). This results in a total ohmic power $P_{\Omega}<350 \mathrm{~W}$ in a filament with $5 \mathrm{~mm}$ radius. It is noted that for the ohmic filaments in the case of oblique pellet injection, the lower value of $T_{\mathrm{e}}$ brings the ohmic power in the filament up to $1 \mathrm{~kW}$.

The ECH power is more difficult to estimate, since the filaments move in and out of 
the heating zone. A conservative estimate is obtained by assuming that the ECH power is evenly distributed over the region inside the $q=1$ radius, and that a filament gets a fraction of the power in proportion to its relative cross section. Thus, a filament with $5 \mathrm{~mm}$ radius inside a $q=1$ region with a radius of $40 \mathrm{~mm}$, sees an average $\mathrm{ECH}$ power density of $p_{\text {fil,ECH }}=20 \mathrm{MW} \mathrm{m}^{-3}$, resulting in a total absorbed power in the filament of $P_{\text {fil,ECH }}=5 \mathrm{~kW}$.

In the absence of a more sophisticated model, we only compute the energy confinement time and the average electron thermal conductivity of a filament in cylindrical geometry. Convection or time dependent terms are neglected in this analysis. For a typical filament in a centrally heated plasma $\left(5 \mathrm{~mm}\right.$ radius, amplitude $600 \mathrm{eV}, n_{\mathrm{e}}=2.5 \times 10^{19} \mathrm{~m}^{-3}$ $P_{\text {fil,ECH }}=5 \mathrm{~kW}$ ), assuming a uniform power density in the filament and a parabolic $T_{\mathrm{e}}$ profile we find $\chi_{\mathrm{fil}, \mathrm{PB}}=0.04 \mathrm{~m}^{2} \mathrm{~s}^{-1}$. This value is an order of magnitude lower than the values normally found for the central region in RTP, which ties in with the fact that inside a magnetic island the magnetic topology is 'good'.

The relevant energy confinement time is associated with the amplitude of the filament, $\tau_{\mathrm{E}, \text { fil }}=\int \frac{3}{2} n_{\mathrm{e}} k\left(T_{\mathrm{e}}-T_{\mathrm{e}, 0}\right) \mathrm{d} V / P_{\text {fil }}$, where $T_{\mathrm{e}, 0}$ is the electron temperature at the foot of the filament, and the integral is taken over the volume of the filament. For the typical ECH filament, we find $\tau_{\mathrm{E}, \text { fil }}=120 \mu \mathrm{s}$. This value compares well with the observation that filaments reappear within $250 \mu$ s after a sawtooth crash. The resistive skin time of a filament, estimated at $\tau_{\mathrm{R}, \text { fil }}=200 \mu \mathrm{s}$, is not much longer than the energy confinement time, thanks to the low thermal diffusivity. In particular, $\tau_{\mathrm{R} \text {,fil }}$ is sufficiently close to $\tau_{\mathrm{E} \text {,fil }}$ to allow the current density to follow the temperature rise in a filament. Thus, it is conceivable that filaments are the result of a thermal instability.

We compare the experimental value of the diffusivity $\chi_{\text {fil,PB }}$ to the neo-classical diffusivity $\chi_{\text {neo. }}$ For the typical ECH filament conditions, the plasma is well within the banana regime $\left(v^{*} \ll 1\right)$ for $\rho>0.01$. In the filament region, i.e. $\rho=0-0.3$, we find $\chi_{\text {neo }}=0.02-0.03 \mathrm{~m}^{2} \mathrm{~s}^{-1}$. The width of the banana orbits is typically several millimetres, due to the low value of the poloidal field in the proximity of the plasma centre. Hence, the experimental value $\chi_{\text {fil, } \mathrm{PB}}$ is quite close to the neo-classical estimate, exceeding it by only a factor up to two. A point of consideration is the fact that the filament diameter is only a few banana widths. This may have an effect on the applicability of the neo-classical transport coefficient, but a treatment of this issue is outside the scope of this paper.

Finally, the question is addressed as to whether the occurrence of filamentation has an impact on the global energy confinement of the plasma. First, it is observed that the filamented volume is so small that it contributes little to the total stored energy. Second, the global energy confinement of RTP discharges with central ECH that show filamentation is in good agreement with the ITER89 scaling. Hence, there is no indication that filamentation is associated with poor confinement.

The filamented region itself is bounded by steep gradients, thanks to which the net confinement in this region is good. This is particularly clear in the case of the filaments which appear in an ohmic discharge during the non-local heating effect. In this case the insulation of the central region can be significantly better than is the case in a normal ohmic discharge. The filamented region itself obviously cannot be described by a one-dimensional transport model.

\section{Acknowledgments}

This work was performed under the Euratom-FOM association agreement, with financial support from NWO and Euratom. The authors would like to thank the RTP-team for very efficient operation of the machine and diagnostics. Furthermore, we would like to thank Franco Porcelli and Enrico Rossi for supplying us with figure 14. 


\section{References}

[1] Chirikov B V 1979 Phys. Rep. 52265

[2] de Vries P C, Waidmann G, Krämer-Flecken A, Donné A J H Donné and Schüller F C 1997 Plasma Phys. Control. Fusion 39 439-51

[3] de Vries P C, Donné A J H, Heijnen S H, Hugenholz C A J, Krämer-Flecken A, Schüller F C and Waidmann G 1997 Nucl. Fusion 37 1641-6

[4] Yamauchi T, Grek B, Hoshino K, LeBlanc B, Felt J, Shiina T, Kurita G, Ishige Y and Kozawa T 1996 Phys. Lett. A 223 179-85

[5] Nave M F F, Edwards A W, Hirsch K, Hugon M, Jacchia A, Lazzaro A, Salzmann H and Smeulders P 1992 Nucl. Fusion 32825

[6] Beurskens M N A, Barth C J, Lopes Cardozo N J and van der Meiden H J 1999 Plasma Phys. Control. Fusion 41 1321-48

[7] Günter S, Gude A, Maraschek M, Sesnic S, Zohm H and ASDEX upgrade team 1998 Nucl. Fusion 38 1431-4

[8] Zou X L, Colas L, Paume M, Chareau J M, Laurent L, Devynck P and Gresillon D 1995 Phys. Rev. Lett. 751090

[9] Colas L, Mou X L, Paume M, Chareau J M, Guiziou L, Hoang G T, Michelot Y and Grésillon D 1998 Nucl. Fusion 38 903-18

[10] Entrop I, Lopes Cardozo N J, Jaspers R and Finken K H 2000 Phys. Rev. Lett. 84 3606-9

[11] Barth C J, Beurskens M N A, Chu C C, Donné A J H, Lopes Cardozo N J, Herranz J, Meiden H J v d and Pijper F J 1997 Rev. Sci. Instrum. 683380

[12] Lopes Cardozo N J, Schüller F C, Barth C J, Chu C C, Pijper F J, Lok J and Oomens A A M 1994 Phys. Rev. Lett. $73256-9$

[13] Lopes Cardozo N J, Schüller F C, Barth C J, Chu C C, Pijper F J, Lok J, Montvai A, Oomens A A M, Peters M and de Rover M 1994 Plasma Phys. Control. Fusion 36 B133-B142

[14] Cima G, Gentle K W, Wootton A, Brower D L, Zeng L, Deng B H, Domier C W and Luhmann N C Jr 1998 Plasma Phys. Control. Fusion 40 1149-58

[15] Mirnov S V 1998 Plasma Phys. Rep. 24 813-17

[16] Porcelli F, Rossi E, Cima G and Wootton A 1999 Phys. Rev. Lett. 82 1458-61

[17] Beurskens M N A, Barth C J, Chu C C and Lopes Cardozo N J 1999 Rev. Sci. Instrum. 70 1999-2011

[18] Donné A J H 1994 Plasma Phys. Rep. 20192

[19] Lopes Cardozo N J et al 1997 Plasma Phys. Control. Fusion 39 B303-B316

[20] Hogeweij G M D et al 1996 Phys. Rev. Lett. 76 632-5

[21] de Baar M R, Hogeweij G M D, Lopes Cardozo N J, Oomens A A M and Schüller F C 1997 Phys. Rev. Lett. 78 4573

[22] Gentle K W et al 1995 Phys. Rev. Lett. 743620

[23] Mantica P et al 1997 Proc. 24th EPS Conf. on Controlled Fusion and Plasma Physics (Berchtesgaden) Part IV, pp 1853-6

[24] Mantica P, Galli P, Gorini G, Hogeweij G M D, de Kloe J, Lopes Carodozo N J and RTP Team 1999 Phys. Rev. Lett. 825048

[25] Mantica P et al 1998 Proc. 25th EPS Conf. on Controlled Fusion and Plasma Physics (Prague)

[26] Hogeweij G M D, Lopes Cardozo N J, de Baar M R and Schilham A M R 1998 Nucl. Fusion 381881

[27] Callen J D and Kissick M W 1997 Plasma Phys. Control. Fusion 39 B173

[28] de Rover M 1996 Chaos in magnetized plasmas Thesis University of Technology, Eindhoven

[29] Kuvshinov B N, Westerhof E and Schep T J 1998 Generation of Electron Vortices by Local Heating, Proc. Joint Varenna-Lausanne Int. Workshop on 'Theory of Plasma' (Varenna, Italy, 31 August-4 September 1998)

[30] Kuvshinov B N, Westerhof E and Schep T J 1998 Electron MHD of Pressure Driven Perturbations in Strongly Magnetized, Inhomogeneous Plasma, Int. Congress on Plasma Physics and ICPP/25th EPS Conf. on Controlled Fusion and Plasma Physics (Prague, Czech Republic, 29 June-3 July 1998) 